\title{
DEFORMATIONS OF THE MODULAR GROUP AS A QUASIFUCHSIAN CORRESPONDENCE
}

\author{
SHAUN BULLETT
}

\begin{abstract}
When viewed as a $(2: 2)$ holomorphic correspondence on the Riemann sphere, the modular group $P S L_{2}(\mathbb{Z})$ has a moduli space $\mathcal{Q}$ of nontrivial deformations for which the limit set remains a topological circle. This space is analogous to a Bers slice of the deformation space of a Fuchsian group as a Kleinian group, but there are certain differences. A Bers slice contains a single quasiconformal conjugacy class of Kleinian groups: we show that for an open dense set of parameter values in $\mathcal{Q}$ the correspondence belongs to a single quasi-conformal conjugacy class, but that at a countable set $\mathcal{C}$ of isolated parameter values it satisfies an additional critical relation. We classify these relations, propose 'pleating coordinates' for $\mathcal{Q}$, and investigate how the correspondence degenerates on the boundary of $\mathcal{Q}$. In particular, we show that there is a point on the boundary of $\mathcal{Q}$ where the correspondence degenerates into a mating between $P S L_{2}(\mathbb{Z})$ and the quadratic polynomial $z \rightarrow z^{2}+1 / 4$. A key ingredient in our analysis is a bijection between $\mathcal{Q} \backslash \mathcal{C}$ and an intermediate cover between the moduli space of the space of non-critical grand orbits of the correspondence, and its universal cover, the corresponding Teichmüller space.
\end{abstract}

\section{The Modular group as a QUASIFUCHSIAN CORRESPONDENCE}

The modular group $P S L_{2}(\mathbb{Z})$ acts on the Riemann sphere $\hat{\mathbb{C}}$ by Möbius transformations

$$
z \rightarrow \frac{a z+b}{c z+d} .
$$

It is well known that the two elements

$$
S: z \rightarrow-\frac{1}{z} \text { and } T: z \rightarrow z+1
$$

together generate $P S L_{2}(\mathbb{Z})$. As an abstract group, $P S L_{2}(\mathbb{Z})$ is the free product $C_{2} * C_{3}$ of the cyclic group of order 2 generated by $S$ and the cyclic group of order 3 generated by $S T$.

Notation. When dealing with the group $C_{2} * C_{3}$ rather than the particular representation $P S L_{2}(\mathbb{Z})$, we shall denote generators of $C_{2}$ and $C_{3}$ by $\sigma$ and $\rho$.

We can parameterise the representations of $C_{2} * C_{3}$ in $P S L_{2}(\mathbb{C})$, up to conjugacy by elements of $P S L_{2}(\mathbb{C})$, by the cross-ratio between the fixed points of $\sigma$ on $\hat{\mathbb{C}}$ and

Received by the editors July 7, 2010.

2010 Mathematics Subject Classification. Primary 37F05; Secondary 37F30.

The author thanks the Heilbronn Institute, University of Bristol, for its support during the writing of this paper, and the Fields institute, Toronto, for providing an ideal environment for early work in March 2006, on the ideas presented here.

(C)2010 American Mathematical Society Reverts to public domain 28 years from publication 
those of $\rho$. Thus there is a one (complex) dimensional moduli space of representations. Within this one-dimensional space is a subset $\mathcal{D}$ of parameter values for which the representation is faithful and discrete. To fix ideas, normalise $\rho$ to be the rotation through positive angle $2 \pi / 3$ about the origin (with fixed points at the origin and infinity). Representations of $C_{2} * C_{3}$ in $P S L_{2}(\mathbb{C})$ are then determined by the ratio $\alpha / \beta$ of the fixed points of $\sigma$. Since we have a choice as to how to label these fixed points, $\alpha / \beta$ and $\beta / \alpha$ determine the same representation, and we may take their sum as our parameter. By Klein's Combination Theorem, the set $\mathcal{D}$ of parameter values for which the representation is faithful and discrete is the set of parameter values for which there exist fundamental domains for $\rho$ and $\sigma$ whose union covers the whole Riemann sphere. Topologically $\mathcal{D}$ is a punctured closed disc (the puncture point corresponds to $\alpha=\beta$ ). For parameter values in the interior of $\mathcal{D}$ the representations have connected ordinary sets and their limit sets are Cantor sets. The modular group $P S L_{2}(\mathbb{Z})$ lies on the boundary of $\mathcal{D}$ and is determined uniquely by the condition that $\sigma \rho$ be parabolic, with fixed point of multiplier +1 , or equivalently, by the condition that the limit set of the representation be a topological circle, separating two completely invariant domains. So there are no non-trivial deformations of the representation $P S L_{2}(\mathbb{Z})$ of $C_{2} * C_{3}$ which keep the limit set a topological circle.

However, as we shall show below, there is a natural way in which the orbits of the representation $P S L(2, \mathbb{Z})$ of the group $C_{2} * C_{3}$ may be regarded as those of a holomorphic correspondence, that is to say a multivalued map $z \rightarrow w$ defined by a polynomial relation $p(z, w)=0$, and in this context there exists a non-trivial space of deformations keeping the limit set a topological circle. We shall completely classify correspondences which lie in the interior of the moduli space of deformations, and make conjectures concerning correspondences which lie on the boundary of this space. A preliminary version of some of the ideas presented here appeared in the final section of [1], but the underlying theory has been substantially developed since then and the reader is warned that there have also been some changes in notation (in particular for the patterns described in Section 6 of the current paper).

We first introduce a class of 'elementary' holomorphic correspondences, analogous to finite groups, which we can combine to create correspondences with more complicated dynamical behaviour. Given any rational map $Q$ of degree $d$, we define the covering correspondence $\operatorname{Cov}^{Q}$ of $Q$ to be the $(d: d)$ correspondence on $\hat{\mathbb{C}}$ given by

$$
z \rightarrow w \Leftrightarrow Q(z)=Q(w) .
$$

We define the deleted covering correspondence $\operatorname{Cov}_{0}^{Q}$ of $Q$ to be the $(d-1: d-1)$ correspondence given by

$$
z \rightarrow w \Leftrightarrow \frac{Q(z)-Q(w)}{z-w}=0 .
$$

This has graph obtained from that of $\operatorname{Cov}^{Q}$ by removing the component of the identity $(w=z)$. Thus for $Q_{0}(z)=z^{3}$ the $(2: 2)$ correspondence $\operatorname{Cov}_{0}^{Q_{0}}$ sends $z$ to $\left\{\rho(z), \rho^{-1}(z)\right\}$, where $\rho(z)=e^{2 \pi i / 3} z$ (Figure 1). Given $\alpha$ and $\beta$ on the positive real axis, let $\sigma$ be the Möbius involution which has these as its fixed points. If $\alpha$ and $\beta$ are chosen such that $\sigma \rho$ and $\sigma \rho^{-1}$ each have just one fixed point (in Figure 1 these are the points $A$ and $B$ respectively), then both $\sigma \rho$ and $\sigma \rho^{-1}$ are parabolic and the 


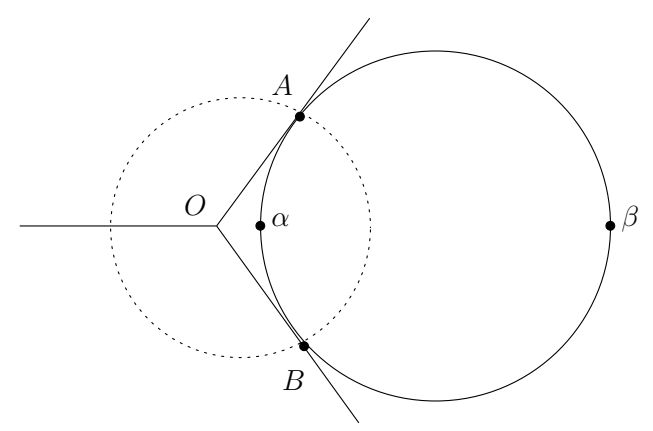

Figure 1. The modular group as a correspondence $\sigma \circ \operatorname{Cov}_{0}^{Q_{0}}:$ the dotted circle is the limit set.

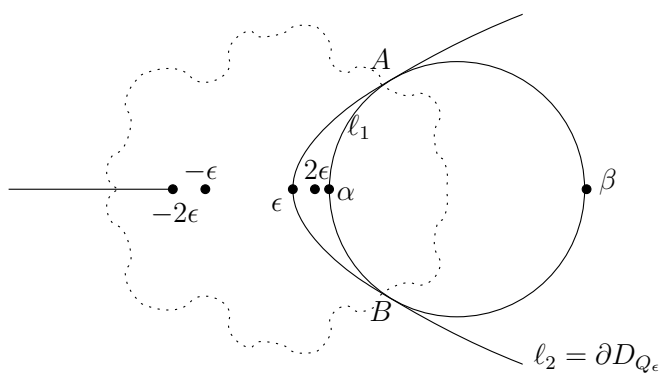

Figure 2. The correspondence $\sigma \circ \operatorname{Cov}_{0}^{Q_{\epsilon}}$ : the limit set (sketched schematically as a dotted line) is still a Jordan curve, but is no longer a round circle.

orbits of $\sigma \circ \operatorname{Cov}_{0}^{Q_{0}}$ are those of a subgroup of $P S L_{2}(\mathbb{C})$ conjugate to $P S L_{2}(\mathbb{Z})$. In particular, the correspondence $\sigma \circ \operatorname{Cov}_{0}^{Q_{0}}$ has a round circle as its limit set.

We may deform the cyclic group $C_{3}=\left\{1, \rho, \rho^{-1}\right\}$ as a correspondence by deforming $Q_{0}$ to $Q_{\epsilon}(z)=z^{3}-3 \epsilon^{2} z$. The effect is to split the double critical point $z=0$ of $Q_{0}$ into a pair of single critical points $z= \pm \epsilon$ : the point $z=\infty$ remains a double critical point. To simplify the description we shall assume at first that $\epsilon$ is real and positive.

The behaviour of $\operatorname{Cov}_{0}^{Q_{\epsilon}}$ is no longer that of a group, though it still has a fundamental domain, for example the region $D_{Q_{\epsilon}}$ to the right of the arc $\ell_{2}$ passing through $z=+\epsilon$ in Figure 2, By a fundamental domain, we mean a maximal subset of $\widehat{\mathbb{C}}$ on which $Q_{\epsilon}$ is injective, in other words a transversal for the branched covering map $Q_{\epsilon}$. The transversal in the figure arises as follows. Consider the inverse image under $Q_{\epsilon}$ of the segment of the negative real axis between the critical values $-\infty$ and $-2 \epsilon^{3}$ of $Q_{\epsilon}$. This inverse image is the set $[-\infty,-2 \epsilon] \cup \ell_{2}$, where $\ell_{2}$ is a smooth curve through the critical point $\epsilon$, as illustrated in Figure 2. The region $D_{Q_{\epsilon}}$ to the right of $\ell_{2}$ has as its two images under $\operatorname{Cov}_{0}^{Q_{\epsilon}}$ the intersection of $\hat{\mathbb{C}} \backslash D_{Q_{\epsilon}}$ with the upper and lower half-planes: the correspondence $\operatorname{Cov}_{0}^{Q_{\epsilon}}$ maps $D_{Q_{\epsilon}}$ to each of these regions by opening up a slit from $z=+\epsilon$ to $z=+2 \epsilon$, mapping one edge of this slit to the interval $[-2 \epsilon,-\epsilon]$ and the other edge to the interval $[-\epsilon,+\epsilon]$; the arc $\ell_{2}$ itself is folded at $+\epsilon$ and mapped to the interval $(-\infty,-2 \epsilon]$. Note that at $\infty$ the 
correspondence $\operatorname{Cov}^{Q_{\epsilon}}$ behaves as a pair of rotations through angles $\pm 2 \pi / 3$. As $\epsilon$ tends to zero the behaviour of $\operatorname{Cov}_{0}^{Q_{\epsilon}}$ near the origin also approaches that of a pair of rotations through angles $\pm 2 \pi / 3$.

If we now choose an involution $\sigma$ which has the property that the circle $\ell_{1}$ through its fixed points touches the curve $\ell_{2}$ (at the points $A$ and $B$ in Figure 2), or equivalently such that the correspondence $\sigma \circ \operatorname{Cov}^{Q_{\epsilon}}$ has two double fixed points, we may expect the limit set of the correspondence to remain a topological circle, and the action of the correspondence outside this topological circle to be conformally conjugate to the action of $P S L_{2}(\mathbb{Z})$ on the complex upper half plane. We shall show that this is indeed the case, investigate how far this behaviour persists as we move further away from the group case by increasing the size of $\epsilon$, and makes conjectures about how it eventually breaks down. Note that up to conformal conjugacy we have just one (complex) degree of freedom in our family of correspondences $\sigma \circ \operatorname{Cov}^{Q_{\epsilon}}$. By rescaling $z$ we may normalise $Q_{\epsilon}$ to $Q_{1}$, and we may make a free choice for the position of one of the fixed points, say $\alpha$, of $\sigma$, but then the condition that the correspondence $\sigma \circ \operatorname{Cov}^{Q_{1}}$ has two double fixed points determines the position of $\beta$ up to a finite number of possibilities (in fact two, as we shall see in the next section) and hence determines $\sigma$ up to the same number of possibilities.

Our interest is in those members of our family of $(2: 2)$ holomorphic correspondences $\mathcal{F}=\sigma \circ \operatorname{Cov}^{Q_{\epsilon}}$ which possess the following properties:

(i) The action of $\mathcal{F}$ on the Riemann sphere is properly discontinuous except on a topological circle $\Lambda$. (To say that $\mathcal{F}$ is properly discontinuous means that each neighbourhood has only finitely many distinct returns under (mixed) iteration of $\mathcal{F}$ and $\mathcal{F}^{-1}$; see 4 for details.)

(ii) The action of $\mathcal{F}$ on $\Lambda$ is topologically conjugate to that of the correspondence $z \rightarrow w$ defined by the relation $(w-T z)\left(w-S T^{-1} S z\right)=0$ on $\hat{\mathbb{R}}=\mathbb{R} \cup \infty$. (The orbits of this correspondence are those of the modular group.)

(iii) The action of $\mathcal{F}$ on one component, denoted $\Omega_{1}$, of the ordinary set $\Omega=\hat{\mathbb{C}} \backslash \Lambda$, is conformally conjugate to that of the correspondence $(w-T z)\left(w-S T^{-1} S z\right)=0$ (the modular group) on the complex upper half-plane.

We shall see in due course that these conditions imply that the topological circle $\Lambda$ of (i) is a quasicircle (the image of a round circle on $\hat{\mathbb{C}}$ under a quasiconformal homeomorphism of $\hat{\mathbb{C}}$ ). It is natural to refer to holomorphic correspondences satisfying (i) and (ii) as quasifuchsian deformations of the modular group. We may view correspondences which satisfy (iii) as well as (i) and (ii) as matings between a deformation of the modular group as a fuchsian correspondence, acting on one component of $\Omega$, and the modular group itself acting on the other. Thus the region in moduli space where our correspondences satisfy conditions (i), (ii) and (iii) is analogous to a Bers slice of the moduli space of a quasifuchsian Kleinian group [8]. However, we shall see that there are some differences - for example our analogue of a Bers slice contains in its interior a countable infinity of parameter values where there are critical relations.

We introduced the correspondences $\sigma \circ \operatorname{Cov}^{Q_{\epsilon}}$ as a family of potential examples. Before proceeding any further, we prove that this family contains a conformal conjugate of every holomorphic correspondence $\mathcal{F}$ having the properties (i), (ii) and (iii) listed above.

Definition 1. We say the holomorphic correspondences $\mathcal{F}, \mathcal{F}^{\prime}$ are conformally conjugate if there is a Möbius transformation $M$ such that $\mathcal{F}^{\prime}=M^{-1} \circ \mathcal{F} \circ M$. 
Proposition 1. Every (2:2) holomorphic correspondence satisfying (i), (ii) and (iii) is conformally conjugate to a correspondence $\mathcal{F}$ of the form $\sigma \circ C_{0 v}{ }^{Q_{\epsilon}}$ where $\epsilon=0$ or $1, Q_{\epsilon}$ is the function $Q_{\epsilon}(z)=z^{3}-3 \epsilon^{2} z$, and $\sigma$ is an involution such that $\mathcal{F}$ has two double fixed points.

Proof. Let $\mathcal{F}$ be a $(2: 2)$ correspondence satisfying (i), (ii) and (iii), and let $\Gamma(\mathcal{F})$ denote the graph of $\mathcal{F}$ (the pairs $(z, w) \in \hat{\mathbb{C}} \times \hat{\mathbb{C}}$ such that $w \in \mathcal{F}(z)$ ). Let $I_{-}$ and $I_{+}$denote the covering involutions for the two projections $p_{-}:(z, w) \rightarrow z$ and $p_{+}:(z, w) \rightarrow w$ from $\Gamma(\mathcal{F})$ to $\hat{\mathbb{C}}$. Condition (iii) ensures that $\left(I_{+} I_{-}\right)^{3}$ is the identity on the part of $\Gamma(\mathcal{F})$ covering the component $\Omega_{1}$ of $\Omega(\mathcal{F})$, and hence, by analytic continuation, that $\left(I_{+} I_{-}\right)^{3}$ is the identity on the whole of $\Gamma(\mathcal{F})$. It now follows that $\sigma: z \rightarrow p_{+}\left(I_{+} I_{-} I_{+}\left(p_{-}^{-1}(z)\right)\right)$ is a well-defined map from $\hat{\mathbb{C}}$ to $\hat{\mathbb{C}}$ (that is to say both values of $p_{-}^{-1}(z)$ give the same value for $\left.\sigma(z)\right)$. Moreover, since $\sigma^{2}$ is the identity on $\Omega_{1}$, it is the identity on the whole of $\hat{\mathbb{C}}$, by analytic continuation. Also $(\sigma \circ \mathcal{F}) \cup I$ is an equivalence relation (where $I$ denotes the identity), again by analytic continuation, using the fact that it is an equivalence relation on $\Omega_{1}$. But the projection map $P$ from points of $\hat{\mathbb{C}}$ to equivalence classes, being holomorphic, is a rational map. Since on $\Omega_{1}$ the correspondence $(\sigma \circ \mathcal{F}) \cup I$ is conjugate to a pair of rotations through angles $\pm 2 \pi / 3$, this map $P$ has a double critical point and hence there exist Möbius transformations $M_{1}$ and $M_{2}$ such that $M_{2} P M_{1}$ is either the polynomial $Q_{0}(z)=z^{3}$ or the polynomial $Q_{1}(z)=z^{3}-3 z$ (depending on whether $P$ has two double critical points or one double and two simple critical points). Thus $\mathcal{F}$ is conjugate to $\sigma \circ \operatorname{Cov}^{Q_{0}}$ or to $\sigma \circ \operatorname{Cov}^{Q_{1}}$. As to the fact that $\mathcal{F}$ must have two double fixed points, this follows at once from property (ii).

Remark 1. The proposition above holds with only properties (i) and (ii) as hypotheses, provided we allow a general rational function $Q$ of degree three in place of the polynomials $Q_{0}$ and $Q_{1}$. For $\left(I_{+} I_{-}\right)^{3}$ to be the identity on $\Gamma(\mathcal{F})$ it is enough that it be the identity on an infinite subset, and in our case it is the identity on $\Gamma\left(\left.\mathcal{F}\right|_{\Lambda}\right)$ by (ii). Similar reasoning applies in the other parts of the proof where arguments concerning analytic continuation are applied.

Remark 2. When $\mathcal{F}$ is a holomorphic correspondence satisfying (i), (ii) and (iii), we refer to $\Lambda$ as the limit set of $\mathcal{F}$. There is no single concept of limit set that will make sense for a general holomorphic correspondence - the difficulty is that the various equivalent conditions that characterise the limit set of a Kleinian group are no longer equivalent to one another for correspondences (see [5] for a discussion). However, for $\mathcal{F}$ satisfying conditions (i), (ii) and (iii), one can show that $\Lambda$, which we defined as the complement of the ordinary set $\Omega$, can also be characterised as the accumulation set of each grand orbit of $\mathcal{F}$.

\section{TwO NORMALISATIONS AND AN ALGEBRAIC RELATION}

We already have one normal form for our family of candidate correspondences, namely $Z \rightarrow W$, where

$$
W \in \sigma \circ \operatorname{Cov}_{0}^{Q_{1}}(Z)
$$

in other words, where

$$
Z^{2}+Z(\sigma(W))+(\sigma(W))^{2}=3
$$


and $\sigma$ is an involution having fixed points (say) $\alpha$ and $\beta$. Thus

$$
\sigma(Z)=\frac{(\alpha+\beta) Z-2 \alpha \beta}{2 Z-(\alpha+\beta)} .
$$

For the correspondence to be quasifuchsian, $\alpha$ and $\beta$ must satisfy a certain algebraic relation, corresponding to the geometric condition that the correspondence has two double fixed points (or one quadruple fixed point). We shall identify this relation shortly.

An alternative normal form is given by taking a new coordinate $z$, where

$$
Z=\frac{\alpha z+\beta}{z+1}
$$

Now the involution $\sigma$ becomes $z \rightarrow-z$, with fixed points at 0 and $\infty$, and the correspondence becomes $z \rightarrow w$, where

$$
\left(\frac{\alpha z+\beta}{z+1}\right)^{2}+\left(\frac{\alpha z+\beta}{z+1}\right)\left(\frac{\alpha w-\beta}{w-1}\right)+\left(\frac{\alpha w-\beta}{w-1}\right)^{2}=3 .
$$

Finally, to bring notation into line with that of 4 , we may replace the parameters $\alpha$ and $\beta$ by new parameters $a=\alpha / \beta$ and $k=1 / \beta^{2}$ and write the correspondence as

$$
\left(\frac{a z+1}{z+1}\right)^{2}+\left(\frac{a z+1}{z+1}\right)\left(\frac{a w-1}{w-1}\right)+\left(\frac{a w-1}{w-1}\right)^{2}=3 k
$$

We shall use whichever of the coordinates $Z$ or $z$, and whichever of the parameter pairs $(\alpha, \beta)$ or $(a, k)$, is most convenient for a particular computation, but we shall employ a capital $Z$ or small $z$ to remind us which coordinate system we are using.

To find when the correspondence (2) has two double fixed points (or one quadruple fixed point), we observe that setting $w=z$ in (2) yields a quadratic equation in $z^{2}$, and that this quadratic equation has a single solution if and only if $\alpha$ and $\beta$ satisfy the relation:

$$
(\alpha+\beta)^{2}-16 \alpha \beta+48=0 .
$$

Note that if $\alpha$ is real, this relation yields a real value for $\beta$ if and only if $|\alpha| \geq 1$. Conversely, if $\beta$ is real, it yields a real value for $\alpha$ if and only if $|\beta| \geq 1$. Relation (4) translates in $(a, k)$ coordinates to the relation

$$
k=1-\frac{(a-7)^{2}}{48}
$$

and the condition that both $\alpha$ and $\beta$ lie in the real interval $[+1, \infty]$ translates into the condition that $a$ is real and lies in the interval $[7,7+4 \sqrt{ } 3]$ or the interval $[7-4 \sqrt{ } 3,1 / 7]=[1 /(7+4 \sqrt{ } 3), 1 / 7]$. Note that $a=7$ corresponds to $k=1$ and that $a=7+4 \sqrt{ } 3$ corresponds to $k=0$ (the modular group case).

Notation $\mathcal{F}_{a}$ for the correspondence. From now on we denote the correspondence $z \rightarrow w$ of the form (3), with parameter values $(a, k)$ satisfying condition $(5)$, by $\mathcal{F}_{a}$.

Exchanging $\alpha$ with $\beta$ corresponds to replacing $a$ by $1 / a$ in $(3)$; indeed, $\mathcal{F}_{a}$ is conjugate to $\mathcal{F}_{1 / a}$ via, by the conjugacy, $z \rightarrow 1 / z$. Conversely:

Lemma 1. The conformal conjugacy class of $\mathcal{F}_{a}$ determines the value of the parameter a uniquely up to $a \sim 1 / a$. 
Proof. In the normalisation (1) the correspondence has singular points at $-1,+1$ and $\infty$, and the only conformal automorphism compatible with this marking is $z \rightarrow-z$. The fixed points $\{\alpha, \beta\}$ of $\sigma$ are also determined by the correspondence, so it is apparent that any change in $\alpha$ or $\beta$ other than an exchange of labels $(\alpha \leftrightarrow \beta)$ or a change of signs $((\alpha, \beta) \leftrightarrow(-\alpha,-\beta)$, induced by the change of coordinates $z \leftrightarrow-z)$ alters the conformal conjugacy class of $\sigma \circ \operatorname{Cov}_{0}^{Q_{1}}$. Since an exchange of labels changes $a$ to $1 / a$, and a change of signs has no effect on $a(=\alpha / \beta)$, the result follows.

\section{REAL CORRESPONDENCE DEFORMATIONS OF THE MODULAR GROUP}

The case that the orbits of the correspondence are (up to conjugacy) those of the modular group $P S L_{2}(\mathbb{Z})$, occurs when $\epsilon=0$ in the normalisation $\sigma \circ \operatorname{Cov}_{0}^{Q_{\epsilon}}$, or equivalently when $\alpha$ and $\beta$ tend to $\infty$ in the normalisation $\sigma \circ \operatorname{Cov}_{0}^{Q_{1}}$ (equation (1)), or equivalently when the correspondence is $\mathcal{F}_{a}$ with $a=7 \pm 4 \sqrt{ } 3$ (and so $k=0$ ). We now consider the dynamics of $\sigma \circ \operatorname{Cov}_{0}^{Q_{1}}$ when $\alpha$ and $\beta$ are both real and strictly greater than 2 , in other words when we are dealing with a 'small' real perturbation from the group case.

When $\alpha$ and $\beta$ are real and $\geq 1$, the two double fixed points of $\sigma \circ \operatorname{Cov}_{0}^{Q_{1}}$ are a complex conjugate pair: they are the points $A$ and $B$ where the $\operatorname{arc} \ell_{2}$ bounding the region $D_{Q_{1}}$ touches the circle centred on the real axis which passes through $\alpha$ and $\beta$ (Figure 2). Note that when $\alpha=1$ we have $\beta=7$ (by relation (4)) and the fixed point $Z=1$ of the correspondence then has multiplicity four.

Proposition $2(9])$. When $\alpha$ and $\beta$ are real, both strictly greater than 2 , and satisfy relation (4) of Section 2, then the correspondence $\sigma \circ C_{0} v_{0}^{Q_{1}}$ is a quasifuchsian deformation of the modular group (in the sense of Section 11).

Proof. See 9] for details, but for convenience we sketch an outline here.

Consider the round disc $D_{\sigma}$ bounded by the circle passing through $\alpha$ and $\beta$ which has centre on the real axis (Figure 3). Its image $\operatorname{Cov}^{Q_{1}}\left(D_{\sigma}\right)$ consists of a pair of (no longer round) discs $D_{1}, D_{2}$ arranged as shown in the figure so that each of $D_{\sigma}$, $D_{1}$ and $D_{2}$ touches the other two. Thus $\sigma \circ \operatorname{Cov}^{Q_{1}}\left(D_{\sigma}\right)$ consists of a pair of discs $D_{3}=\sigma\left(D_{2}\right)$ and $D_{4}=\sigma\left(D_{1}\right)$ touching at $\sigma(C)$ as shown. Images $\left(\sigma \circ \operatorname{Cov}^{Q_{1}}\right)^{n}\left(D_{\sigma}\right)$ of $D_{\sigma}$ for $n>1$ yield nested families of discs inside $D_{3}$ and $D_{4}$. The action of the correspondence is properly discontinuous except at the set $\Lambda$ of points which lie in infinitely many of these discs or in the $\sigma$-images of infinitely many of them. There are two kinds of infinite intersections of nests of discs to consider: those where every point in the intersection is an interior point of every disc in the nest, and those which contain boundary points of discs. By applying the Grötzsch inequality one can show that the first type of intersection is a single point, and by analysing the behaviour of the correspondence around its fixed points, one can show that the second type of intersection is also a single point. Finally, by combining these methods, one can show that $\Lambda$ is a topological circle and that the action of the correspondence on it is conjugate to that of the modular group on $\hat{\mathbb{R}}$.

A fundamental domain for the action of the correspondence $\sigma \circ \operatorname{Cov}_{0}^{Q_{1}}$ on $\Omega=$ $\hat{\mathbb{C}} \backslash \Lambda$ is given by $D_{Q_{1}} \backslash D_{\sigma}$. This has two components, $\Delta_{1}$ (containing $Z=\infty$ ), and $\Delta_{2}$ (containing $Z=1$ ): these are fundamental domains for the action of the correspondence on the two components $\Omega_{1}$ and $\Omega_{2}$ of $\Omega$. The only critical or cocritical point of $Q_{1}$ in $\Omega_{1}$ is at $Z=\infty$, which is a double critical point, and thus 


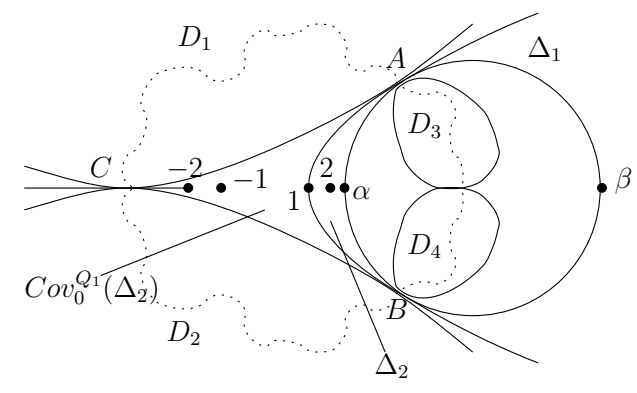

Figure 3 . The disc $D_{\sigma}$ (bounded by the circle through $\alpha, \beta, A$ and $B$ ) and its images $D_{1}, D_{2}, D_{3}$ and $D_{4}$, discussed in the proof of Proposition 2 ,

$\operatorname{Cov}^{Q_{1}}$ acts on $\Omega_{1}$ as a pair of rotations $\left\{\rho, \rho^{-1}\right\}$, fixing $Z=\infty$ and such that $\rho^{3}=I$. It follows at once by the Klein Combination Theorem that $\sigma \circ \operatorname{Cov}^{Q_{1}}$ acts on $\Omega_{1}$ as a faithful discrete representation of $C_{2} * C_{3}$, with $\Delta_{1}$ as a fundamental domain. That this representation is conjugate to the repesentation of $C_{2} * C_{3}$ on the upper half-plane as the modular group now follows from the fact that the action has limit set homeomorphic to $\hat{\mathbb{R}}$.

Still restricting attention to the case that both fixed points $\alpha, \beta$ of $\sigma$ are real, we next consider what happens when we allow one of them to take values $\leq 2$. When $\alpha=2$ (and so $\beta=26$, and when the correspondence is written $\mathcal{F}_{a}$, the value of $a$ is $a_{1}=13$ ) we encounter our first critical relation. The points -2 and +2 now lie on the same grand orbit of $\sigma \circ \operatorname{Cov}_{0}^{Q_{1}}$ and the topology of Figure 3 changes: the boundaries of the discs $D_{1}$ and $D_{2}$ now touch the real axis at $Z=-1$ as well as at the point labelled $C$. As we decrease $\alpha$ below the value 2, the boundaries of the discs $D_{1}$ and $D_{2}$ touch along an interval containing $Z=-1$ : in effect $D_{1}$ and $D_{2}$ fuse together to form a single disc, the boundary of which has a 'selfcontact' point at $C$. Further decreases give a sequence of critical relations and corresponding topological changes to fundamental domains, but, as is shown in [9], the correspondence continues to satisfy conditions (i), (ii) and (iii) for all positive real values of $\alpha>1$. We list the real critical coincidence values in Theorem 1 below. It is convenient to introduce some notation first, so that we can label various points without reference to a particular coordinate system.

Notation for critical and singular points. We denote the forward singular points of $\mathcal{F}_{a}$ by $p_{1}$ and $p_{2}$, that is to say $p_{1}$ and $p_{2}$ are the points which have one image rather than two under $\mathcal{F}_{a}$. In the normalisation (1) they are the co-critical points of $Q_{1}$; thus $p_{1}$ is the point $Z=-2$ and $p_{2}$ is the point $Z=+2$. We denote the corresponding critical points of $Q_{1}$ by $c_{1}(Z=+1)$ and $c_{2}(Z=-1)$. The backward singular points of $\mathcal{F}_{a}$ are denoted by $q_{1}=\sigma\left(p_{1}\right)$ and $q_{2}=\sigma\left(p_{2}\right)$ : these are the points which have just one image under $\mathcal{F}_{a}^{-1}$.

Theorem 1. (i) On the segment $[7,7+4 \sqrt{ } 3]$ of the real axis in the space of the parameter $a$, there exist:

- $a$ unique value $a_{0}=7+4 \sqrt{ } 3$ at which $p_{1}=p_{2}$;

- for each positive integer $n$, a unique value $a_{n}$ at which $q_{2}$ maps to $p_{2}$ under $n-1$ iterations of $\mathcal{F}_{a}$; 
- a unique value $a_{\infty}=7$ at which a forward orbit of $q_{2}$ under $\mathcal{F}_{a}$ is asymptotic to the fixed point $\alpha$ of $\sigma$.

(ii) When $n$ is even, the grand orbits of $p_{1}$ and $p_{2}$ under $\mathcal{F}_{a_{n}}$ coincide. When $n$ is odd, the grand orbits of $p_{2}$ and $\alpha$ coincide, and when $n=\infty$ the grand orbits of $p_{1}$ and $\alpha$ coincide.

Proof. (i) At the value $a_{0}=7+4 \sqrt{ } 3$ the correspondence is conjugate to the modular group. Here various marked points on the real $z$-axis become identified and we see them laid out in the order

$$
p_{1}=c_{2}=c_{1}=p_{2}<\alpha<q_{2}=\sigma\left(c_{1}\right)=\sigma\left(c_{2}\right)=q_{1} .
$$

Note that the parameter value $a_{0}$ corresponds to the limiting case $\alpha=\beta=\infty$ in the $Z$-coordinate system.

Decreasing $a$ below $a_{0}$, the fixed points $\alpha$ and $\beta$ of the involution $\sigma$ lie on the real $Z$-axis and the order of the marked points here (or on the real $z$-axis) becomes

$$
p_{1}<c_{2}<c_{1}<p_{2}<\alpha<q_{2}<\sigma\left(c_{1}\right)<\sigma\left(c_{2}\right)<q_{1} .
$$

The next critical coincidence is at the value $a_{1}=13$, where the middle three points in the order coincide, $p_{2}=\alpha=q_{2}$. Then for $a$ just below $a_{1}$, the order of the middle three points is reversed, to $q_{2}<\alpha<p_{2}$. At $a_{2}$ the point $q_{2}$ coincides with $c_{1}$ (and so of course $p_{2}$ coincides with $\sigma\left(c_{1}\right)$ ). When $a$ descends below $a_{2}$, the order of the points becomes:

$$
p_{1}<c_{2}<q_{2}<c_{1}<\alpha<\sigma\left(c_{1}\right)<p_{2}<\sigma\left(c_{2}\right)<q_{1} .
$$

The relevant orbit of $q_{2}$ is generated by the branch of $\sigma \circ \mathrm{Cov}^{Q}$ that can be described as a 'reflection in $c_{1}$ followed by a reflection in $\alpha$ '. This composite is a translation to the right and it follows by an easy monotonicity argument that as $a$ decreases the number of steps taken for the orbit of $q_{2}$ to pass $p_{2}$ becomes increasingly large. Thus each value $a_{n}$ of the statement occurs exactly once, as $a$ descends from $a_{0}=7+4 \sqrt{ } 3$ to $a_{\infty}=7$.

(ii) We note that for $n=2 m+1$ (with $m \geq 0$ ), $a_{n}$ may be defined to be the (unique) real value of $a>7$ at which the backward singular point $q_{2}$ maps to the fixed point $\alpha$ of $\sigma$ under $m$ iterations of $\mathcal{F}_{a}$. As $\sigma$ conjugates $\mathcal{F}_{a}$ to $\mathcal{F}_{a}^{-1}$, it follows by symmetry under $\sigma$ that $q_{2}$ maps to $p_{2}$ under $2 m=n-1$ iterations of $\mathcal{F}_{a}$. Also, for $n=2 m$ (with $m \geq 1$ ), $a_{n}$ may be defined to be the (unique) real value of $a>7$ at which $q_{2}$ maps to the critical point $c_{1}$ under $m-1$ iterations of $\mathcal{F}_{a}$. Since $\mathcal{F}_{a}$ always sends $c_{1}$ to $\sigma\left(c_{1}\right)$, it follows by symmetry under $\sigma$ that $q_{2}$ maps to $p_{2}$ under $2 m-1=n-1$ iterations of $\mathcal{F}_{a}$. For $n=\infty$, it is easily seen that $c_{1}=\alpha$.

A series of three computer plots in the $z$-plane, for decreasing real values of $a$, is shown in Figure 4. In each picture the lines plotted are the images of the boundary of the disc $D_{\sigma}$ and $\partial D_{Q_{1}}$ (in red and blue respectively). In the right-hand plot, where $\alpha=1$ and $\beta=7$, or equivalently when $a=a_{\infty}=7$ and $k=1$ in the normalisation (3), we find that the limit set $\Lambda$ has been pinched at a single point to form a 'figure of eight', dividing the component $\Omega_{2}$ of the ordinary set into two discs, restricted to which the correspondence is respectively a $(2: 1)$ correspondence conjugate to the 'cauliflower quadratic' $q_{1 / 4}: z \rightarrow z^{2}+1 / 4$ on its filled Julia set $K\left(q_{1 / 4}\right)$ and a $(1: 2)$ correspondence conjugate to $q_{1 / 4}^{-1}$ on $K\left(q_{1 / 4}\right)$. This is the correspondence described as a mating between $P S L_{2}(\mathbb{Z})$ and $q_{1 / 4}$ in $[4$. We shall return to this example in the final section of this paper. 

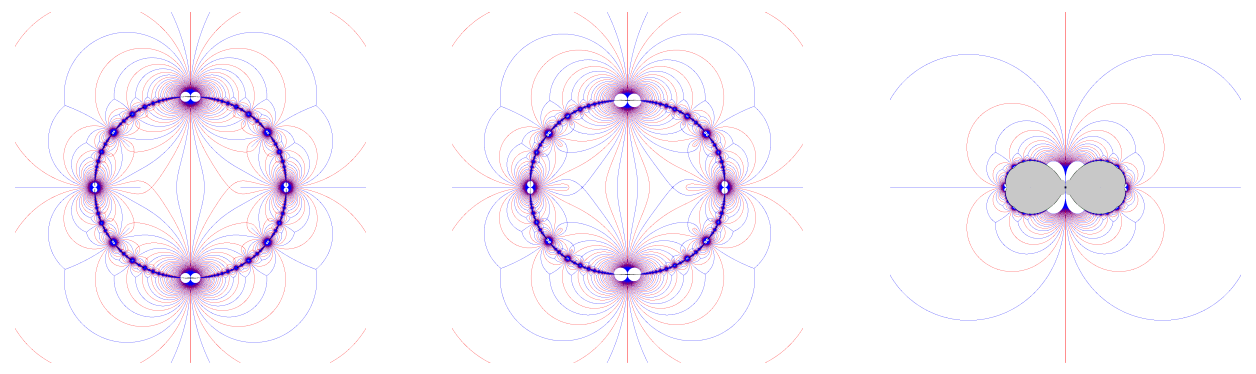

Figure 4. Computer plots illustrating the dynamics of $\mathcal{F}_{a}$ for the parameter values $a_{1}, a_{2}$ and $a_{\infty}$ (see Theorem 11). In the plot for $a=a_{\infty}$, restricted to the left-hand grey disc as domain and codomain the correspondence $\mathcal{F}_{a}$ is conjugate to $q_{1 / 4}(z)=z^{2}+1 / 4$ on its filled Julia set $K\left(q_{1 / 4}\right)$; restricted to the right-hand grey disc it is conjugate to $\left(q_{1 / 4}\right)^{-1}$ on $K\left(q_{1 / 4}\right)$.

\section{Behaviour of THE CORRESPONDENCE FOR COMPLEX VAlueS OF THE PARAMETER}

Experimental observation suggests that the plane of the parameter $a$ can be divided up into a number of regions corresponding to different types of behaviour of the correspondence $\mathcal{F}_{a}$. The situation is illustrated schematically in Figure 5 . Recall that to pass to moduli space we must identify $a$ with $1 / a$ : under this identification the regions labelled $I$ and $I V$ are identified, the region labelled $I I I$ is quotiented by an involution fixing $a=1$, and the region $I I$ is quotiented by an involution fixing $a=-1$.

Regions $I$ and $I V$ are where the correspondence $\mathcal{F}_{a}$ appears to be a quasifuchsian deformation of the modular group, in the sense defined in Section 1 These regions will be our main concern. However we digress briefly to discuss the geometry and dynamics of $\mathcal{F}_{a}$ when $a$ lies in region $I I I$. For $a$ real in the interval $1<a<7$ the fixed points $A$ and $B$ of the correspondence become a pair of real numbers rather than a pair of complex conjugates, and they are no longer points of contact between the boundary circle of $D_{\sigma}$ and the boundary $\ell_{2}$ of the region $D_{Q_{1}}$ : the disc $D_{\sigma}$ is now strictly contained within $D_{Q_{1}}$, and we have a geometric setup which determines that the correspondence is a mating between a quadratic polynomial $q_{c}: z \rightarrow z^{2}+c$ and a discrete faithful representation of $C_{2} * C_{3}$, 3, 4. The condition that the fixed points of the correspondence be a pair of double points translates into the condition that $q_{c}$ has just one fixed point other than $\infty$, and hence that $c=1 / 4$. Region $I I I$ is the set of parameter values where $\mathcal{F}_{a}$ is such a mating. This region is punctured at $a=1$ (where $\mathcal{F}_{a}$ becomes degenerate) and double covers the moduli space of all matings between $q_{1 / 4}$ and discrete faithful representations of $C_{2} * C_{3}$. On the boundary of region $I I I$ we conjecture [2] that there is a dense set of 'cusps', one for each rational $0 \leq p / q<1$, corresponding to the circle-packing representations of $C_{2} * C_{3}$ on the boundary of the moduli space of discrete faithful representations of $C_{2} * C_{3}$. For parameter values in the region $I I$ (complementary to $I, I I I$ and $I V)$ it appears that every grand orbit of the correspondence is dense on the Riemann sphere. 


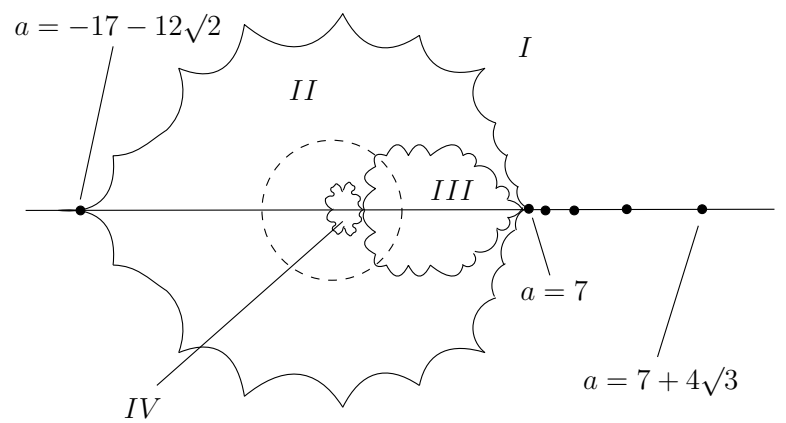

Figure 5. Schematic picture of the plane of the parameter $a$. The unit circle is drawn as a dotted curve. The correspondence $\mathcal{F}_{a}$ is a quasifuchsian deformation of the modular group when $a$ is in region $I$ or region $I V$ (these regions map to one another under $a \leftrightarrow 1 / a)$.

\section{ThE SPACE OF ORBITS OF A QUASIFUCHSIAN CORRESPONDENCE}

We shall denote by $\mathcal{Q}$ the moduli space of quasifuchsian correspondences, that is to say the space of conformal equivalence classes of quasifuchsian correspondences, in the sense of Section 1, Formally we define $\mathcal{Q}$ to be a subset of the quotient of the Riemann sphere parameterised by $a$ under the identification $a \sim 1 / a$.

However, since the experimental evidence suggests that there are no values of $a$ on the unit circle for which $\mathcal{F}_{a}$ is quasifuchsian, we shall abuse notation and regard $\mathcal{Q}$ as a subset of $\{a: a \in \hat{\mathbb{C}},|a|>1\}$. In other words we shall regard $\mathcal{Q}$ as region $I$ of Figure 5 together with $\infty$. This is merely a notational convenience: the sceptical reader may replace all occurrences of ' $a$ ' by 'the equivalence class of $a$ under $a \sim 1 / a^{\prime}$ in what follows.

Remark 3. We allow the value $a=\infty$, since although when written in the form (3) the correspondence $\mathcal{F}_{a}$ becomes degenerate at $a=\infty$, this is just an artefact of the normalisation and $\alpha=(4 \sqrt{ } 3) i, \beta=0$ is a solution to relation (4) which yields a perfectly good quasifuchsian correspondence.

For $a \in \mathcal{Q}$, the ordinary set $\Omega$ of the correspondence $\mathcal{F}_{a}$ is a disjoint union of two components: $\Omega_{1}$, containing the double fixed point of $\operatorname{Cov}_{0}^{Q_{1}}$ (the point $Z=\infty$ ), and $\Omega_{2}$, containing the two critical points $c_{1}$ and $c_{2}$ of $Q_{1}$ together with the corresponding co-critical points (the forward singular points $p_{1}$ and $p_{2}$ of $\mathcal{F}_{a}$ ). As before we use the notation $q_{1}$ and $q_{2}$ for the backward singular points $\sigma\left(p_{1}\right)$ and $\sigma\left(p_{2}\right)$ of $\mathcal{F}_{a}$. Note that $\sigma$ is a branch of $\mathcal{F}_{a} \circ \mathcal{F}_{a}^{-1} \circ \mathcal{F}_{a}$, so for each of $i=1,2$ the points $p_{i}$ and $q_{i}$ are always on the same grand orbit of $\mathcal{F}_{a}$.

For every correspondence $\mathcal{F}_{a}$ with $a \in \mathcal{Q}$ the components $\Omega_{1} /\left\langle\mathcal{F}_{a}\right\rangle$ and $\Omega_{2} /\left\langle\mathcal{F}_{a}\right\rangle$ of the grand orbit space $\Omega /\left\langle\mathcal{F}_{a}\right\rangle$ have the following structure:

(i) $\Omega_{1} /\left\langle\mathcal{F}_{a}\right\rangle$ is a Riemann surface homeomorphic to a sphere, with two marked points and a cusp. The marked points correspond to the orbits of the fixed points of the generators $\rho$ and $\sigma$ of $P S L_{2}(\mathbb{Z})$ acting on the upper half plane, and the cusp corresponds to the fixed point of $\sigma \rho$ (on the real axis). 
(ii) $\Omega_{2} /\left\langle\mathcal{F}_{a}\right\rangle$ is a Riemann surface homeomorphic to a sphere with a cusp and three marked points $P_{1}, P_{2}$ and $Q$ (corresponding to the orbits of the singular points $p_{1}$ and $p_{2}$ and the fixed point $\alpha$ of $\left.\sigma\right)$.

Definition 2. We say that the correspondence $\mathcal{F}_{a}$, for $a \in \mathcal{Q}$, satisfies a critical relation if at least two of the three marked points in the grand orbit space $\Omega_{2} /\left\langle\mathcal{F}_{a}\right\rangle$ coincide, that is to say either

(a) $p_{1}$ is on the same grand orbit as $p_{2}$, or

(b) the fixed point of $\sigma$ lies on the grand orbit of $p_{1}$ or of $p_{2}$.

We denote by $\mathcal{C}$ the set of parameter values in $\mathcal{Q}$ where critical relations occur.

Let $\mathcal{M}$ denote the moduli space of complex structures on once-punctured spheres equipped with a marked pair of distinct points $\left\{P_{1}, P_{2}\right\}$ (where it is not specified which point is which) and a marked point $Q$ distinct from the first two. Thus:

$$
\mathcal{M}=\left(\left((\hat{\mathbb{C}})^{4}-\operatorname{Diag}\right) / P S L_{2}(\mathbb{C})\right) / C_{2}=\left(\left((\hat{\mathbb{C}})^{4}-\operatorname{Diag}\right) / C_{2}\right) / P S L_{2}(\mathbb{C}) .
$$

Here Diag denotes the 'thick' diagonal, $P S L_{2}(\mathbb{C})$ acts diagonally on $(\hat{\mathbb{C}})^{4}$, and $C_{2}$ acts by interchanging the middle two entries in a 4-tuple. For every $a \in \mathcal{Q} \backslash \mathcal{C}$ the orbit space $\Omega_{2} /\left\langle\mathcal{F}_{a}\right\rangle$ determines a point $\hat{\pi}(a) \in \mathcal{M}$ by regarding the puncture point, the marked pair of points $\left\{P_{1}, P_{2}\right\}$ and the marked point $Q$ as a 4 -tuple of elements of $\hat{\mathbb{C}}$.

For any $a \in \mathcal{Q} \backslash \mathcal{C}$ we may deform the complex structure on the orbit space of $\mathcal{F}_{a}$ in any way we please, and obtain a new correspondence quasiconformally conjugate to $\mathcal{F}_{a}$. This new correspondence is conformally conjugate to $\mathcal{F}_{a^{\prime}}$ for some $a^{\prime} \in \mathcal{Q} \backslash \mathcal{C}$ (by Proposition 11). Thus $\hat{\pi}: \mathcal{Q} \backslash \mathcal{C} \rightarrow \mathcal{M}$ is a surjection.

A correspondence $\mathcal{F}_{a}$ with $a \in \mathcal{Q} \backslash \mathcal{C}$ determines a point of $\mathcal{M}$, but to reconstruct $\mathcal{F}_{a}$ from a point of $\mathcal{M}$, that is to say from a complex structure on $\Omega_{2} /\left\langle\mathcal{F}_{a_{b}}\right\rangle$ where $a_{b}$ is some chosen base point of $\mathcal{Q} \backslash \mathcal{C}$, we need the additional information as to which 'tiles' (sheets of the branched covering) contain the singular points of $\mathcal{F}_{a}$. To organise this information coherently, it is convenient first to pass to a double cover of $\mathcal{Q} \backslash \mathcal{C}$. Let $(\mathcal{Q} \backslash \mathcal{C})^{\prime}$ denote the space of pairs $\left(a, p_{1}\right)$, where $a \in \mathcal{Q} \backslash \mathcal{C}$ and $p_{1}$ is one of the two singular points of $\mathcal{F}_{a}$. Thus $(\mathcal{Q} \backslash \mathcal{C})^{\prime}$ is the unramified double covering of $\mathcal{Q} \backslash \mathcal{C}$ corresponding to the two ways the labels $p_{1}, p_{2}$ can be attached to the singular points of $\mathcal{F}_{a}$.

The projection from $\mathcal{Q} \backslash \mathcal{C}$ to $\mathcal{M}$ is covered by a projection from $(\mathcal{Q} \backslash \mathcal{C})^{\prime}$ to the moduli space of 4 distinct marked points on the Riemann sphere, that is:

$$
\mathcal{M}^{\prime}=\left((\hat{\mathbb{C}})^{4}-\operatorname{Diag}\right) / P S L_{2}(\mathbb{C}) \cong \hat{\mathbb{C}} \backslash\{0,1, \infty\} .
$$

The projection $\hat{\pi}^{\prime}: \mathcal{M}^{\prime} \rightarrow \mathcal{M}$ (allowing the interchange of the middle two entries in a 4-tuple) is a double cover ramified at the 4-tuple $(0,1,-1, \infty) \in \mathcal{M}^{\prime}$, since there is Möbius transformation sending $(0,1,-1, \infty)$ to $(0,-1,1, \infty)$. The covering involution of $\hat{\pi}^{\prime}$ fixes the ramification point and one of the three puncture points of $\mathcal{M}^{\prime} \cong \hat{\mathbb{C}} \backslash\{0,1, \infty\}$, and exchanges the other two puncture points. Thus $\mathcal{M}$ is isomorphic to the punctured plane $\mathbb{C}^{*}=\mathbb{C} \backslash\{0\}$.

Different points $a \in \mathcal{Q} \backslash \mathcal{C}$ may yield grand orbit spaces $\Omega_{2} /\left\langle\mathcal{F}_{a}\right\rangle$ with the same complex structure. In order to reconstruct a unique correspondence $\mathcal{F}_{a}$ from its grand orbit space, we shall need some form of marking on this orbit space. 

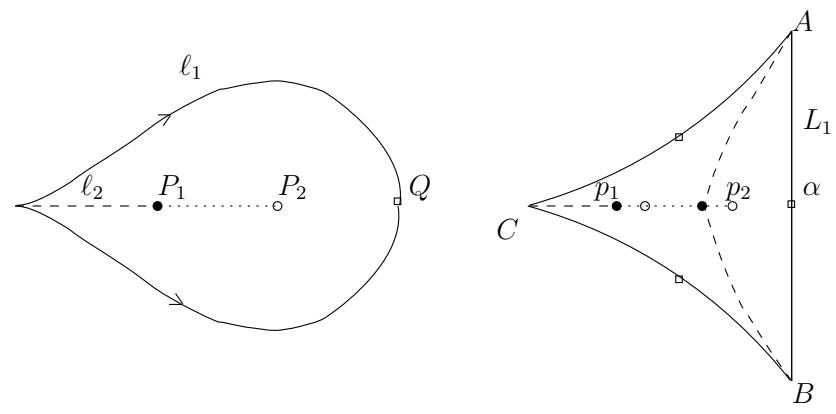

FiguRE 6 . On the left: the punctured sphere $\Omega_{2} /\left\langle\mathcal{F}_{a}\right\rangle$ (the arrowed edges are identified). On the right: a lift of $\Omega_{2} /\left\langle\mathcal{F}_{a}\right\rangle$ to a fundamental tile $\Delta$ for $\mathcal{F}_{a}$.

Definition 3. For $a \in \mathcal{Q} \backslash \mathcal{C}$, a fundamental tile for $\mathcal{F}_{a}=\sigma \circ C o v^{Q_{1}}$ is any triangle $\Delta \subset \Omega_{2}$ with the properties:

(i) $\Delta$ is invariant under $\operatorname{Cov}^{Q_{1}}$.

(ii) The fixed points $A$ and $B$ of $\mathcal{F}_{a}$ are vertices of $\Delta$.

(iii) The edge $A B$ of $\Delta$ contains the fixed point $Q$ of $\sigma$ and this edge is invariant under $\sigma$.

(iv) The interior of $\Delta$ contains the critical and co-critical points of $\operatorname{Cov}^{Q_{1}}$.

The triangle $\Delta=\Delta_{2} \cup \operatorname{Cov}_{0}^{Q_{1}}\left(\Delta_{2}\right)$ in Figure 3 is an example of a fundamental tile. The domain $\Omega_{2}$ is tiled by copies of $\Delta$ which are mapped to one another by branches of $\left(\sigma \circ \operatorname{Cov}^{Q_{1}}\right)^{n}(n \in \mathbb{Z})$ in exactly the same way as in the case of the modular group, so the information provided by a fundamental tile and the positions of the critical points $p_{1}$ and $p_{2}$ of $Q_{1}$ on it allows us to reconstruct the correspondence.

A fundamental tile for $\mathcal{F}_{a}$ triple covers (with ramification) the grand orbit space $\Omega_{2} /\left\langle\mathcal{F}_{a}\right\rangle$. We shall define a marking on the grand orbit space which is sufficient to provide a fundamental tile together with the positions of $p_{1}$ and $p_{2}$ and a labelling for these points.

Definition 4. A point of the Teichmüller space $\mathcal{T}$ consists of a point of $\mathcal{M}^{\prime}$ together with an isotopy class of a pair of non-intersecting paths, namely:

(i) a path $\ell_{1}$ from the puncture point to $Q$, and

(ii) a path $\ell_{2}$ from the puncture point to $P_{1}$.

The Teichmüller space $\mathcal{T}$ is the universal cover of $\mathcal{M}^{\prime}$. The data provided by an element of $\mathcal{T}$ is sufficent for us to reconstruct a pair $\left(\mathcal{F}_{a}, p_{1}\right)$ with $a \in \mathcal{Q} \backslash \mathcal{C}$ from the grand orbit space of the correspondence: it enables us to draw a fundamantal tile $\Delta$ containing the two singular points as marked points (Figure 6) and hence to construct $\Omega_{2}$ and to define $\left(\mathcal{F}_{a}, p_{1}\right)$.

But the data provided by a point of $\mathcal{T}$ is more than we need to determine a pair $\left(\mathcal{F}_{a}, p_{1}\right)$. Suppose we change $\ell_{1}$ to $\ell_{1}^{\prime}$ and $\ell_{2}$ to $\ell_{2}^{\prime}$. When we lift $\ell_{1}^{\prime}$ and $\ell_{2}^{\prime}$ to $\Omega_{2}$, let $L_{1}^{\prime}$ denote that lift of $\ell_{1}^{\prime}$ which runs from the vertex $A$ of $\Delta$ (a fixed point of the correspondence $\mathcal{F}_{a}$ ) to the fixed point $\alpha$ of $\sigma$. Let $\Delta^{\prime}$ denote the triangle formed by $L_{1}^{\prime} \cup \sigma\left(L_{1}^{\prime}\right)$ and its image under $\operatorname{Cov}_{0}^{Q_{1}}$ : this triangle has the same vertices $A, B, C$ as $\Delta$. 

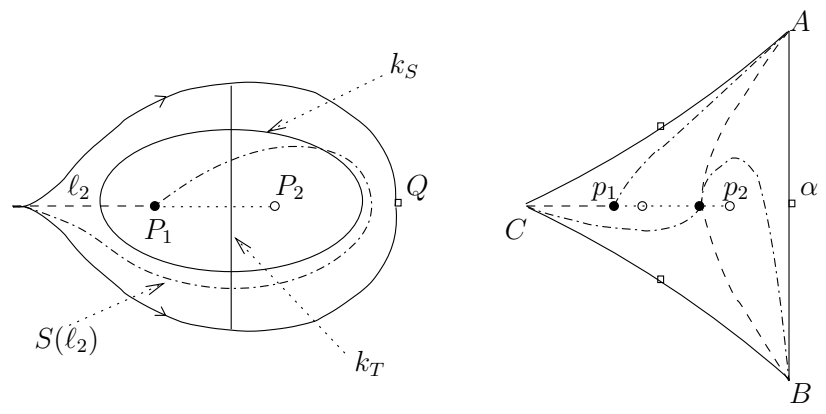

Figure 7. On the left: Dehn twists $S$ and $T$ are about the curves $k_{S}$ and $k_{T}$; the image $S\left(\ell_{2}\right)$ of $\ell_{2}$ is shown dot-dashed. On the right: the lifts (dot-dashed) of $S\left(\ell_{2}\right)$ to $\Delta$.

Lemma 2. $\left(\ell_{1}^{\prime}, \ell_{2}^{\prime}\right) \in \mathcal{T}$ defines the same pair $\left(\mathcal{F}_{a}, p_{1}\right)$ as $\left(\ell_{1}, \ell_{2}\right) \in \mathcal{T}$ if and only if $\left(\ell_{1}^{\prime}, \ell_{2}^{\prime}\right)$ satisfies the following condition:

$\left.{ }^{*}\right)\left\{p_{1}, p_{2}\right\} \subset \Delta^{\prime}$, and the lift of $\ell_{2}^{\prime}$ to $\Delta^{\prime}$ contains an arc from $C$ to $p_{1}$.

Proof. Given an element of $\mathcal{M}^{\prime}$, the extra data that determines $\left(\mathcal{F}_{a}, p_{1}\right)$ is that of which lifts of $P_{1}, P_{2}$ and $Q$ are the singular point $p_{1}$ of the correspondence, the singular point $p_{2}$ of the correspondence and the fixed point $\alpha$ of the associated involution $\sigma$.

The fundamental group $\pi_{1}\left(\mathcal{M}^{\prime}\right)$ of the moduli space $\mathcal{M}^{\prime}$ is generated by a Dehn twist $S$ about the curve $k_{S}$ in Figure 7 and a Dehn twist $T$ about the curve $k_{T}$ in the same figure. Indeed $\pi_{1}\left(\mathcal{M}^{\prime}\right)$ is the free group on these two generators: each word in the group generated by $S$ and $T$ sends the marking $\left(\ell_{1}, \ell_{2}\right)$ to a new marking $\left(\ell_{1}^{\prime}, \ell_{2}^{\prime}\right)$ and all markings are obtained in this way.

Let $\Gamma=\pi_{1}\left(\mathcal{M}^{\prime}\right)$ and let $G$ denote the subgroup of $\Gamma$ consisting of all words $W$ in $S$ and $T$ for which $\left(\ell_{1}^{\prime}, \ell_{2}^{\prime}\right)=\left(W\left(\ell_{1}\right), W\left(\ell_{2}\right)\right)$ satisfy condition $\left(^{*}\right)$ of Lemma 2 ,

Proposition 3. (i) There is a commutative diagram:

$$
\begin{array}{rlll}
\mathcal{T} \stackrel{\pi^{\prime \prime}}{\rightarrow} & \mathcal{T} / G & \stackrel{\pi^{\prime}}{\rightarrow} & \mathcal{T} / \Gamma \\
& \cong \downarrow & & \downarrow \cong \\
& (\mathcal{Q} \backslash \mathcal{C})^{\prime} & \stackrel{\pi^{\prime}}{\rightarrow} & \mathcal{M}^{\prime} \cong \hat{\mathbb{C}} \backslash\{0, \infty\} \\
& \pi \downarrow & & \downarrow \hat{\pi}^{\prime} \\
& \mathcal{Q} \backslash \mathcal{C} & \stackrel{\hat{m}}{\rightarrow} & \mathcal{M} \cong \mathbb{C}^{*}
\end{array}
$$

where $\pi^{\prime \prime}$ and $\pi^{\prime}$ are (unramified) coverings of infinite degree.

(ii) $\pi_{1}(\mathcal{Q} \backslash \mathcal{C})$ acts freely on $\mathcal{T}$ and contains $G$ as a subgroup of index 2.

Proof. Part (i) is an immediate consequence of Lemma2 by standard covering space theory; (ii) follows since $\pi$ is an (unramified) covering of degree two.

Corollary 1. $\hat{\pi}: \mathcal{Q} \backslash \mathcal{C} \rightarrow \mathcal{M}$ is a branched covering. Every branch point in $\mathcal{Q} \backslash \mathcal{C}$ has (local) degree 2 .

Proof. This follows at once from Proposition 3 and the fact that $\hat{\pi}^{\prime}: \mathcal{M}^{\prime} \rightarrow \mathcal{M}$ is a branched cover of degree 2 . 
We identify some specific elements of $G$, and derive some consequences:

Proposition 4. (i) $\left(\ell_{1}^{\prime}, \ell_{2}^{\prime}\right)=\left(S^{3}\left(\ell_{1}\right), S^{3}\left(\ell_{2}\right)\right)$ and $\left(\ell_{1}^{\prime}, \ell_{2}^{\prime}\right)=\left(T^{2}\left(\ell_{1}\right), T^{2}\left(\ell_{2}\right)\right)$ each satisfy condition $(*)$ of Lemma 2 ,

(ii) On a punctured disc neighbourhood of $a_{0}$ (the parameter value where the correspondence is conjugate to the modular group), $\hat{\pi}: \mathcal{Q} \backslash \mathcal{C} \rightarrow \mathcal{M}$ is an unramified covering of degree 3 . On a punctured disc neighbourhood of $a_{1}$ (where the first real critical relation occurs), it is an unramified covering of degree 2 .

Proof. For part (i) in the case of $S$, consider the right hand diagram in Figure 7 . showing the lifts to $\Delta \subset \Omega_{2}$ of the image of $\ell_{2}$ under $S$ : it is only after the third iteration of $S$ that the lift of $\ell_{2}$ starting at $C$ returns to a path linking $C$ to $p_{1}$. When we apply $T$ it is $\ell_{1}$ that moves rather than $\ell_{2}$, and so the triangle $\Delta$ in the lift moves to a new position $\Delta^{\prime}$. One can check that $p_{2}$ lies in $\sigma\left(\Delta^{\prime}\right)$ and not in $\Delta^{\prime}$, but that after two applications of $T$ the shifted triangle contains both $p_{1}$ and $p_{2}$. Part (ii) follows at once from (i).

We next consider the consequences of our analysis of $\mathcal{Q} \backslash \mathcal{C}$ for the quasifuchsian region $\mathcal{Q}$ itself.

Proposition 5. (i) $\mathcal{C}$ is a discrete subset of $\mathcal{Q}$.

(ii) $\hat{\pi}: \mathcal{Q} \backslash \mathcal{C} \rightarrow \mathcal{M}$ extends to a locally ramified cover $\mathcal{Q} \rightarrow(\mathcal{M} \cup\{$ point $\}) \cong \mathbb{C}$.

(iii) $\mathcal{Q}$ is open and path-connected.

(iv) For every $a \in \mathcal{Q}$, the limit set of $\mathcal{F}_{a}$ is a quasicircle.

Proof. (i) This is implicit in our earlier proofs, but to make it explicit we observe that if $\mathcal{F}_{a}$ satisfies a critical relation, then a small perturbation of the parameter $a$ ensures that the projections onto $\Omega_{2} /\left\langle\mathcal{F}_{a}\right\rangle$ of the singular points and the fixed point of $\sigma$ become three distinct points. Thus points of $\mathcal{C}$ are isolated in $\mathcal{Q}$.

(ii) Similar reasoning to part (ii) of Proposition 4 applies to punctured disc neighbourhoods in $\mathcal{Q}$ of all other points of $\mathcal{C}$.

(iii) That $\mathcal{Q}$ is open and path-connected follows from the fact that $\mathcal{T} \rightarrow \mathcal{Q} \backslash \mathcal{C}$ is a branched covering projection and the fact that $\mathcal{T}$ is path-connected.

(iv) Since one can move from any $\mathcal{F}_{a}$ with $a \in \mathcal{Q} \backslash \mathcal{C}$ to any $\mathcal{F}_{a^{\prime}}$ with $a^{\prime} \in \mathcal{Q} \backslash \mathcal{C}$ by a continuous deformation of the complex structure on the grand orbit space of $\mathcal{F}_{a}$, it is immediate that there is a quasiconformal homeomorphism between the limit sets of $\mathcal{F}_{a}$ and $\mathcal{F}_{a^{\prime}}$ : indeed there is an equivariant holomorphic motion on $\hat{\mathbb{C}}$ taking $\mathcal{F}_{a}$ to $\mathcal{F}_{a^{\prime}}$. In a disc neighbourhood of the parameter value $a_{0} \in \mathcal{Q}$, although there can no longer be a holomorphic motion of the whole of $\hat{\mathbb{C}}$ which is equivariant with respect to the correspondences $\mathcal{F}_{a}$ (since $\mathcal{F}_{a_{0}}$ has a critical relation), nevertheless the limit set $\Lambda_{a}$ of $\mathcal{F}_{a}$ moves holomorphically. This follows from the fact that the periodic points of $\mathcal{F}_{a}$ which lie in $\Lambda_{a}$ have expressions which are analytic in $a$ and therefore move holomorphically, the fact that these periodic points are dense in $\Lambda_{a}$, as is observed in the proof of Proposition 2 and the fact that any holomorphic motion of a set extends to its closure [7, 10. But $\Lambda_{a_{0}}$ is a round circle, since the branches of $\mathcal{F}_{a_{0}}$ are conjugate to those of the modular group acting on the upper half-plane. Thus we now know that for $a \in\left\{a_{0}\right\} \cup(\mathcal{Q} \backslash \mathcal{C})$ the limit set of $\mathcal{F}_{a}$ is a quasicircle. The same reasoning we have just applied to a neighbourhood of $a_{0}$ applies to a neighbourhood of any other critical relation parameter value $c \in \mathcal{C}$ : it follows that $\Lambda_{a}$ is a quasicircle for all $a \in \mathcal{Q}$. 
Remark 4. An alternative proof of (iii) follows from the observation that for any parameter value $a$ close to (but not equal to) $a_{0}$ one may modify $\mathcal{F}_{a}$ to $\mathcal{F}_{a_{0}}$ by an equivariant quasiconformal surgery supported on an open set disjoint from the limit set.

Remark 5. It is enlightening to establish the relationship between our moduli spaces and the moduli space of complex structures on once-punctured tori. By its definition, $\mathcal{M}^{\prime}$ is the moduli space of spheres marked at four distinct points. Puncturing the sphere at the first point and double covering it, ramifying at all four marked points, yields a punctured torus. Moreover, every punctured torus with a complex structure comes equipped with a unique involution under which its quotient is a punctured sphere marked at three distinct points. So $\mathcal{M}^{\prime}$ can also be viewed as the moduli space of complex structures on the punctured torus. This close connection between quasifuchsian correspondences and complex structures on the punctured torus will underlie much of what we do in the next section.

\section{PATterns on tiles AND GLOBAL LAMinAtions}

The correspondences associated to parameter values in $\mathcal{Q} \backslash \mathcal{C}$ form a single quasiconformal conjugacy class. We choose a base point $a_{b} \in \mathcal{Q} \backslash \mathcal{C}$, very close to the parameter value $a_{0}$ corresponding to the modular group (which is a point of $\mathcal{C}$ ), and for simplicity we take $a_{b}$ to be real. The correspondence $\mathcal{F}_{a_{b}}$ is conjugate to some $\sigma \circ \operatorname{Cov}_{0}^{Q_{\epsilon}}$, with $\epsilon=\epsilon_{0}$ some fixed small (but non-zero) real value. Choose a marking $\left(\ell_{1}, \ell_{2}\right)$ for the corresponding point of $\mathcal{M}$ and let $\Delta$ denote the fundamental tile in $\Omega_{2}$ corresponding to this marking.

Let $c \in \mathcal{C}$. For $a \in \mathcal{Q} \backslash \mathcal{C}$ sufficiently close to $c$, the two singular points which become identified at the parameter value $c$ are joined by an $\operatorname{arc} \ell_{a}$ in $\Omega_{2}$, which can be chosen so as to project injectively to the grand orbit space $\Omega_{2} /\left\langle\mathcal{F}_{a}\right\rangle$. If we now follow a path in $\mathcal{Q} \backslash \mathcal{C}$ from $a$ to the base point $a_{b}$, deforming $\mathcal{F}_{a}$ to $\mathcal{F}_{a_{b}}$, the arc $\ell_{a}$ is deformed to an $\operatorname{arc} \ell_{a_{b}}$ which projects injectively to $\Omega_{2} /\left\langle\mathcal{F}_{a_{b}}\right\rangle$. Contracting $\ell_{a_{b}}$ to a point corresponds to transforming $\mathcal{F}_{a_{b}}$ to $\mathcal{F}_{c}$. We are therefore led to consider isotopy classes of embedded arcs in $\Omega_{2} /\left\langle\mathcal{F}_{a_{b}}\right\rangle$ which have ends at two of the three marked points.

Given such an embedded arc in $\Omega_{2} /\left\langle\mathcal{F}_{a_{b}}\right\rangle$, consider all its lifts to $\Omega_{2}$. The correspondence $\operatorname{Cov}_{0}^{Q_{\epsilon_{0}}}=\sigma \circ \mathcal{F}_{a_{b}}$, maps the fundamental tile $\Delta$ to itself. Globally the forward and backward iterates $\left(\mathcal{F}_{a_{b}}\right)^{n}(n \in \mathbb{Z})$ of $\mathcal{F}_{a_{b}}$ permute the copies of $\Delta$ in exactly the same manner as the elements of $P S L_{2}(\mathbb{Z})$ in the group case. We call the union of lifts of the arc $\ell_{a_{b}}$ to $\Delta$ a simple invariant pattern. Some examples of such patterns are illustrated in Figure 8. The copies of such a pattern on the images of $\Delta$ fit together to form a global lamination on $\Omega_{2}$, invariant under $\mathcal{F}_{a_{b}}$ (Figure 9). Global laminations obtained in this way are of two types:

Type 1. The leaves are all arcs with end points within $\Omega_{2}$. The lamination on the right in Figure 9 is an example. For such a lamination, contracting the leaves to points corresponds to following a path in $\mathcal{Q} \backslash \mathcal{C}$ from $a_{b}$ to a point of $\mathcal{C}$. Such a contraction can be performed in practice by defining a one-parameter family of ellipse fields on a neighbourhood of each arc, and applying the Measurable Riemann Mapping Theorem.

Type 2. The leaves are all non-compact arcs, each with either one or both ends on the boundary of $\Omega_{2}$. The lamination on the left in Figure 9 is an example. 

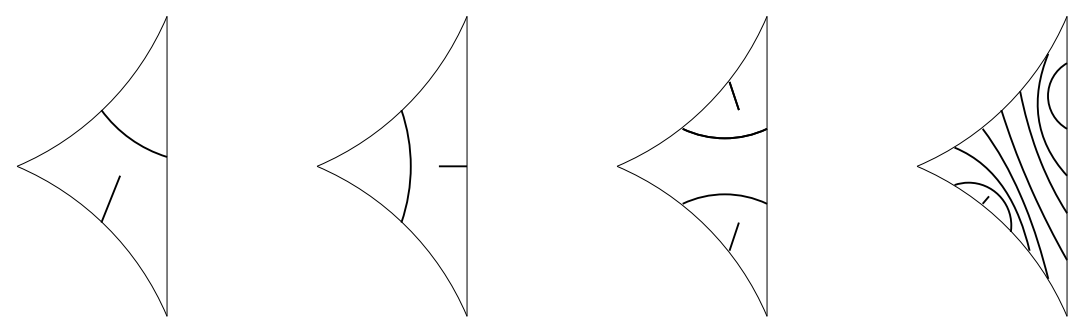

Figure 8. Patterns $P_{1,0}, \rho\left(P_{1,0}\right), P_{1,1}$ and $\rho\left(P_{2,3}\right)$ : the vertical edge is $E_{1}$.
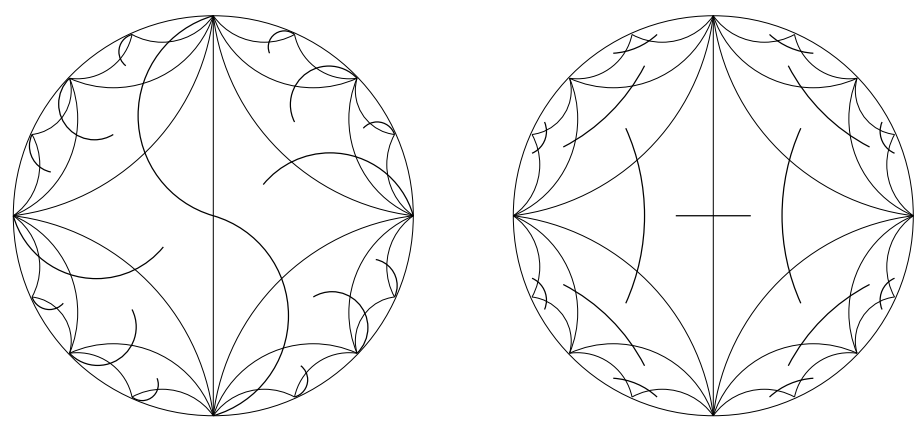

Figure 9. The global laminations corresponding to $P_{1,0}$ and $\rho\left(P_{1,0}\right)$.

We conjecture that, in this case, contracting the leaves to points corresponds to converging to a boundary point of $\mathcal{Q}$, but we do not yet have the technology to prove this.

We set about classifying patterns on tiles and the resulting global laminations. In what follows $\Delta$ is an ideal triangle, invariant under $\operatorname{Cov}_{0}^{Q_{\epsilon_{0}}}$, and a fundamental domain for the action of $\mathcal{F}_{a_{b}}$ on $\Omega_{2}$ (and therefore containing a fixed point of $\sigma$ on one of its edges).

Definition 5. A set $\mathcal{P}$ of non-intersecting $\operatorname{arcs}$ on $\Delta$, each with either one or both end points on $\partial \Delta$, is called a simple invariant pattern if:

(i) the set $\mathcal{P}$ is the lift of a simple arc in $\Omega_{2} /\left\langle\mathcal{F}_{a_{b}}\right\rangle$ joining the images of two of the three singular points $\left(p_{1}, p_{2}\right.$ and the fixed point of $\left.\sigma\right)$;

(ii) every arc in $P$ is 'essential' in the sense that it cannot be removed by an isotopy of $\Omega_{2} /\left\langle\mathcal{F}_{a_{b}}\right\rangle$ keeping the marked points fixed.

We say that two patterns are isotopic if one can be deformed to the other by an isotopy of $\Delta$ sending edges to edges and vertices to vertices.

A simple invariant pattern is mapped $(2: 2)$ to itself by the correspondence $\operatorname{Cov}_{0}^{Q_{\epsilon_{0}}}$, and so, in particular, each side of $\Delta$ must contain the same number of end points of arcs. We denote the three edges of $\Delta$ by $E_{1}, E_{2}$ and $E_{3}$, where $E_{1}$ is the edge containing the fixed point of $\sigma$ and (for definiteness) the edges are labelled anticlockwise. 

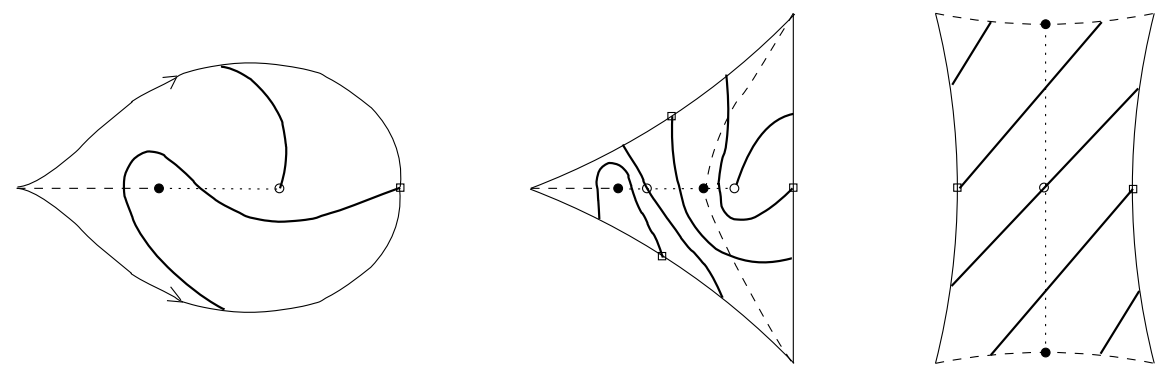

FiguRE 10. An arc on the punctured sphere joining two marked points (left), its lift to the pattern $\rho\left(P_{1,2}\right)$ on the fundamental tile $\Delta$ (centre), and its lift to the geodesic of slope $2 / 3$ on the punctured torus (right).

Lemma 3. For each coprime pair of positive integers $m, n$, and also for $(m . n)=$ $(0,0),(0,1)$ and $(1,0)$, there is a unique simple invariant pattern $P_{m, n}$ which has the following two properties:

(i) $P_{m, n}$ has $m+n$ end points on each edge of $\Delta$.

(ii) The $m+n$ arcs starting on $E_{1}$ all continue to another edge; $m$ of them finish on $E_{2}$ and $n$ of them finish on $E_{3}$.

Proof. This is immediate from the definition. Examples are illustrated in Figure 8. If $m+n$ is odd, then the fixed point of $\sigma$ is an end point, and in this case if $m$ is even, there are $m / 2$ 'loops' with both ends on $E_{3},(n-1) / 2$ loops with both ends on $E_{2}$ and a single arc from $p_{1}$ to $E_{2}$. If $m+n$ is even, then the fixed point of $\sigma$ is not an end point, but as both $m$ and $n$ are then odd, there are $\operatorname{arcs}$ from $p_{1}$ to $E_{2}$ and from $p_{2}$ to $E_{3}$.

Proposition 6. Every simple invariant pattern is of the form $P_{m, n}, \rho\left(P_{m, n}\right)$ or $\rho^{-1} P_{m, n}$ (where $\rho$ denotes rotation through $2 \pi / 3$ ) for $(m, n)$ as in Lemma 3 ,

Proof. A simple invariant pattern descends to a geodesic (or to a union of geodesics) on $\Omega_{2} /\left\langle\mathcal{F}_{a_{b}}\right\rangle$, which in turn lifts to a closed geodesic (or union of closed geodesics) on the punctured torus which double covers $\Omega_{2} /\left\langle\mathcal{F}_{a_{b}}\right\rangle$, ramified at the puncture point and the three marked points. On this torus, marked by the generating curves given by the lifts of our markings on $\Omega_{2} /\left\langle\mathcal{F}_{a_{b}}\right\rangle$, the closed geodesics correspond one-to-one to the rational numbers (Figure 10). Moreover, each closed geodesic on the punctured torus passes through exactly two of the three ramification points and thus gives rise to one of the simple invariant patterns $P_{m, n}, \rho\left(P_{m, n}\right)$ or $\rho^{-1} P_{m, n}$ on $\Delta$.

Remark 6. (i) The various patterns $P_{m, n}, \rho\left(P_{m, n}\right)$ and $\rho^{-1} P_{m, n}$ for $(m, n)$ are distinct for $(m, n)$ with $m+n>1$, but there are obvious identities $\rho\left(P_{0,0}\right)=$ $\rho^{-1}\left(P_{0,0}\right)=P_{0,0}, P_{1,0}=\rho\left(P_{0,1}\right), P_{0,1}=\rho^{-1}\left(P_{1,0}\right)$ and $\rho\left(P_{1,0}\right)=\rho^{-1}\left(P_{0,1}\right)$.

(ii) For future reference we record the explicit correspondence between patterns of the form $\rho\left(P_{m, n}\right)$ and closed geodesics on the punctured torus. With the torus marked as in Figure 10, the pattern $\rho\left(P_{m, n}\right)$ corresponds to the geodesic on the torus of slope $n /(m+n)$, and the pattern $P_{m, n}$ corresponds to the geodesic of slope $(m+2 n) /(m+n)$. 

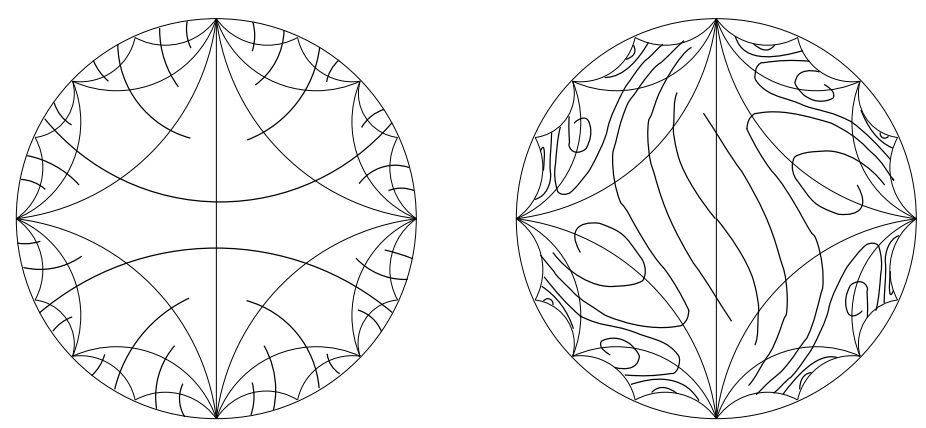

FiguRE 11. The global laminations corresponding to $P_{1,1}$ and $\rho\left(P_{1,2}\right)$.

More examples of simple invariant patterns and associated global laminations are illustrated in Figure 11. These global laminations are obtained by applying words in $\sigma$ and $\rho$ to the fundamental tile in exactly the same way that one would apply them to the corresponding fundamental tile for the modular group. It is easily proved that the global laminations corresponding to patterns $P_{m, n}$ are of Type 2: the leaves which cross the central vertical axis $E_{1}$ are 'infinite' arcs, ending on the boundary of $\Omega_{2}$. The global laminations corresponding to patterns $\rho^{i}\left(P_{m, n}\right)$, with $i= \pm 1$, can be of either Type 1 or Type 2 .

Each element of the group $G=\pi_{1}\left((\mathcal{Q} \backslash \mathcal{C})^{\prime}, a_{b}\right)$, defined in the previous section, sends the fundamental tile $\Delta$ to a new fundamental tile $\Delta^{\prime}$, with the same vertices as $\Delta$ and containing the same marked points of the correspondence $\mathcal{F}_{a_{b}}$. The global lamination on $\Omega_{2}$ is unchanged, but its intersection with $\Delta^{\prime}$ provides a new invariant pattern.

Theorem 2. Under the action of $\pi_{1}\left((\mathcal{Q} \backslash \mathcal{C})^{\prime}, a_{b}\right)$, every simple invariant pattern is equivalent to one of the following:

(i) $P_{m, n}$ for some pair $m, n$;

(ii) $\rho\left(P_{1, n}\right)$ for some $n \geq 0$, or $\rho^{-1}\left(P_{m, 1}\right)$ for some $m \geq 0$.

Proof. Let $P$ be an invariant pattern which has $m+n$ arcs meeting each edge of $\Delta$. If $P$ is not of the form $P_{m, n}$, then by Proposition $\left[\right.$ it has the form $\rho\left(P_{m, n}\right)$ or $\rho^{-1}\left(P_{m, n}\right)$. Assume it has the form $\rho\left(P_{m, n}\right)$ (the other case being completely analogous). Now the top $m$ points of $P \cap E_{1}$ are end points of 'non-traversing' arcs, that is to say arcs which start and end on $E_{1}$, or an arc (at most one) which starts on $E_{1}$ and ends at a singular point in the interior of $\Delta$. The remaining $n$ points of $P \cap E_{1}$ are joined by arcs to $E_{2}$.

Assume that $m>1$ (since if $m=1$ we are done). It will suffice to show that we can replace $\Delta$ by an alternative fundamental domain $\Delta^{\prime}$ for $\mathcal{F}_{a_{b}}$, with the same vertices and containing the same marked points as $\Delta$, but such that the cardinality of the intersection of each edge of $\Delta^{\prime}$ with the lamination is strictly less than $m+n$.

First suppose that $m>n$. Consider the new $\operatorname{arc} E_{1}^{\prime}$ between the vertices $A$ and $B$ of $\Delta$ illustrated in the left-hand part of Figure 12. This is obtained by applying the Dehn twist $T^{2}$ to the image of $E_{1}$ on the grand orbit space $\Omega_{2} /\left\langle\mathcal{F}_{a_{b}}\right\rangle$. The sides $E_{2}^{\prime}$ and $E_{3}^{\prime}$ of $\Delta^{\prime}$ are obtained by applying $\operatorname{Cov}_{0}^{Q}$ to $E_{1}^{\prime}$, and we end up with the new fundamental domain $\Delta^{\prime}$ shown, containing precisely the same marked critical and singular points as $\Delta$. If $m>n$, then $E_{1}^{\prime}$ has smaller cardinality of intersection 

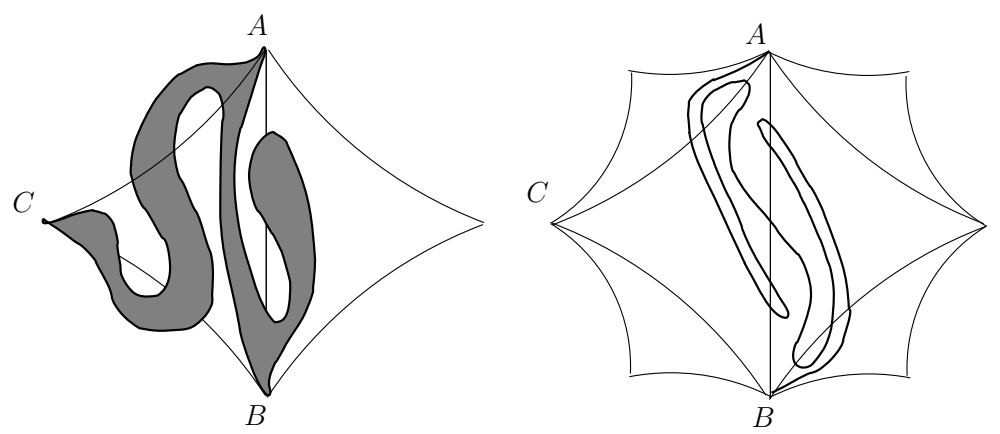

Figure 12. Left: the arc $E_{1}^{\prime}$ from $A$ to $B$ and the fundamental tile $\Delta^{\prime}$ (shaded). Right: the arc $E_{1}^{\prime \prime}$ from $A$ to $B$. (See the proof of Theorem 2, )

with the lamination than does $E_{1}$. We can see this by using the explicit lift to the punctured torus described in Remark 6. The arc $E_{1}^{\prime}$ lifts to the geodesic $\lambda$ of slope $1 / 4$ through the puncture point. On the cylinder obtained by identifying top and bottom sides of the square $\lambda$ has four components. Every geodesic on the cylinder of slope $p / q$ strictly between 0 and $1 / 2$ has the property that all its $q$ components meet $\lambda$ at most once, and at least one of these components is disjoint from $\lambda$ : hence, the intersection of such a geodesic with $\lambda$ has cardinality strictly less than $q$.

If $m<n$, the arc $E_{1}^{\prime}$ from $A$ to $B$ in the left-hand illustration in Figure 12 no longer has the desired property. However, for $1 / 2<n /(n+m)<2 / 3$ the arc $E_{1}^{\prime \prime}$ illustrated in the right-hand part of Figure 12, which corresponds to the geodesic on the torus of slope $7 / 12$, through the puncture point, does the trick. This arc is obtained by applying the composition $T S^{2} T$ of Dehn twists to the image of $E_{1}$ in the grand orbit space. The reader is invited to draw his or her own picture of the fundamental tile $\Delta^{\prime \prime}$ bounded by $E_{1}^{\prime \prime}$ and its images under $\operatorname{Cov}_{0}^{Q}$. Next, for $2 / 3<n /(n+m)<3 / 4$ we use the arc $E_{1}^{\prime \prime \prime}$ corresponding to the geodesic on the torus of slope $17 / 24$ through the puncture point.

To complete the proof, it may verified that:

(i) for each positive integer $q$ the $\operatorname{arc} E_{1}^{(q)}$ corresponding to the geodesic on the torus of slope $1-(1 / q+1 /(q+1)) / 2$ through the puncture point has the property that the corresponding $\Delta^{(q)}$ contains the same marked points as $\Delta$, and

(ii) for every coprime pair $(m, n)$ having $1-1 / q<n /(n+m)<1-1 /(q+1)$, this $E_{1}^{(q)}$ has fewer points of intersection with the pattern $\rho\left(P_{m, n}\right)$ than does $E_{1}$.

\section{The Classification of CORRESPONDENCES HAVING CRITICAL RELATIONS}

In this section we prove that $\mathcal{C}$ consists only of the real parameter values $a_{n}$ listed in Theorem 1 (Section 3). We first formalise what we mean by contracting the global lamination $\Gamma$ (a union of arcs) associated to a pattern $P$. Let $p_{0}(z, w)=0$ be the polynomial relation defining the correspondence $\mathcal{F}_{a_{b}}$.

Definition 6 (2]). A convergent pinching deformation for $\Gamma$ is a family of quasiconformal maps $\left(\phi_{t}\right)_{0 \leq t<1}$ of the Riemann sphere such that the conjugate correspondences $p_{t}$ defined by

$$
p_{t}(z, w)=p_{0}\left(\phi_{t}^{-1}(z), \phi_{t}^{-1}(w)\right)
$$




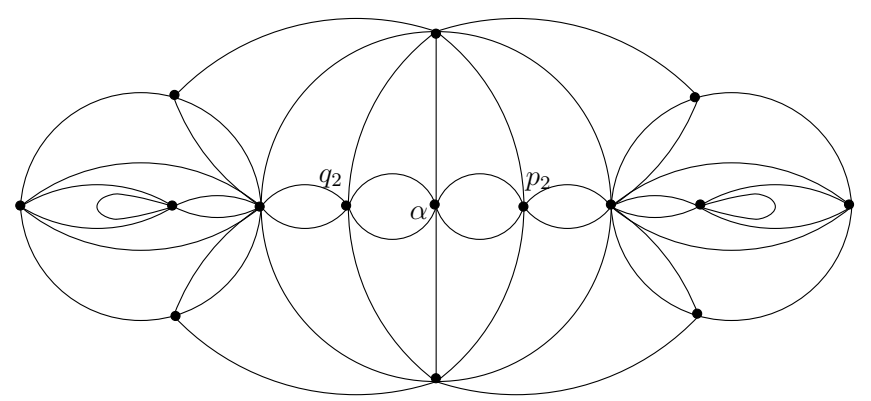

Figure 13. The global lamination associated to $\rho\left(P_{1,2}\right)$ (the pattern on the right in Figure 11), after the leaves of the lamination have been pinched to points.

are holomorphic and satisfy the following:

- $\left(p_{t}, \phi_{t}\right)$ are uniformly convergent to a pair $\left(p_{1}, \phi_{1}\right)$ as $t$ tends to 1 .

- The non-trivial fibres of $\phi_{1}$ are exactly the closure of the connected components of $\Gamma$.

Proposition 7. If the global lamination $\Gamma$ associated to a pattern has leaves which are intervals with end points in the interior of $\Omega_{2}$, then $\Gamma$ supports a pinching deformation converging to a correspondence associated to some parameter value in the interior of $\mathcal{Q}$.

Proof. $\Gamma$ projects to an arc in the grand orbit space. All we need is a neighbourhood of this arc on which to define a one parameter family of ellipse fields giving rise to a convergent pinching deformation. The technical details are as explained in [2] (but the setting here is much simpler).

As all critical relations that occur at interior points of $\mathcal{Q}$ are obtained by contracting laminations satisfying the hypothesis of Proposition 7, it just remains for us to identify the pinched correspondences.

Theorem 3. (i) The global lamination corresponding to $P_{0,0}$ supports a pinching deformation which converges to the real correspondence $\mathcal{F}_{a_{0}}$ of Theorem 1 , that is to say a real correspondence which has grand orbits those of a group conjugate to $P S L_{2}(\mathbb{Z})$.

(ii) For each $n \geq 0$ the global laminations corresponding to the patterns $\rho\left(P_{1, n}\right)$ and $\rho^{-1}\left(P_{n, 1}\right)$ both support pinching deformations converging to the real correspondence $\mathcal{F}_{a_{n+1}}$ of Theorem 1 .

Proof. Statement (i) is self-evident. For (ii) we examine a typical case: $\rho\left(P_{1, n}\right)$ for $n=2$ (proofs for other values of $n$ and for $\rho^{-1}\left(P_{n, 1}\right)$ are analogous). Figure 13 illustrates the global lamination $\rho\left(P_{1,2}\right)$ after pinching: the leaves in Figure 11 have now become dots, but the regions are mapped to one another by the correspondence in exactly the same fashion as that implied by Figure 11. The pinched correspondence is rigid: it has a 0 -dimensional deformation space, since the grand orbit space is a sphere with just two marked points in addition to the puncture point. It is apparent from Figure 13 that this pinched correspondence has a reflection symmetry in the horizontal axis, and thus that it is conjugate to 
one of our $\mathcal{F}_{a}$ with $a \in \hat{\mathbb{R}}$. It is immediate from Figure 11 that under one iteration of the correspondence the leaf containing the backward singular point $q_{2}$ maps to that containing the fixed point point of $\sigma$, so $q_{2}$ maps to $p_{2}$ under two iterations, and hence, by Theorem 1 the pinched correspondence is $\mathcal{F}_{a_{3}}$.

Corollary 2. The only correspondences which satisfy critical relations and whose parameter values lie in the interior of $\mathcal{Q}$ are the real correspondences $\mathcal{F}_{a_{n}}$ (where $0 \leq n<\infty)$ listed in Theorem 1 .

\section{8. 'Pleating RAYS' FOR THE QUASIFUCHSIAN REGION, AND EXAMPLES ON ITS BOUNDARY}

A quasifuchsian once-punctured tous group acting on the Riemann sphere has a quasicircle as its limit set, and a pair of topological discs as its regular set. A Bers slice of the moduli space of such groups consists of groups which have a fixed conformal conjugacy type on one component of the regular set and a variable type on the other. One may assign pleating coordinates (see [6]) to such a Bers slice. These coordinates lift to the corresponding Teichmüller space $\mathcal{T}$ and so provide us (albeit indirectly) with a coordinate system on $\mathcal{Q} \backslash \mathcal{C}$. Taking $a_{b}$ as the base point, pleating rays in $\mathcal{T}$ project to rays in $\mathcal{Q}$, as illustrated in Figure 14.

As one moves along a particular ray, the corresponding arc in $\Omega_{2} /\left\langle\mathcal{F}_{a_{b}}\right\rangle$ is contracted. In $\mathcal{T}$ itself the assignment of pleating coordinates is one-to-one: there is a ray for each $p / q \in \mathbb{Q}$, and these rational rays are dense in $\mathcal{T}$ [6]. But projected onto $\mathcal{Q} \backslash \mathcal{C}$ the labelling of rays emanating from $a_{0}$ is projected from $\mathbb{Q}$ to $\mathbb{Q} / 3 \mathbb{Z}$, so we may choose to label the rays here by rationals $-3 / 2<p / q \leq 3 / 2$. Although the projected rays criss-cross one another after intersecting the real axis (draw the $\left(-\frac{1}{3}\right)$-ray in Figure 14), we conjecture that up to their first crossing of the real axis they remain disjoint and moreover that their union is dense in $\mathcal{Q}$.

Remark 7. 'Pleating coordinates' are assigned to the moduli space of quasifuchsian once-punctured torus groups in a natural way arising from the geometry of the action of such groups as hyperbolic isometries of the Poincaré three-disc: the boundary of the convex hull of the limit set of the group carries a 'pleating' which determines the ray on which the group lies in moduli space 6 . An obvious question is whether there is some analogous geometric definition of 'pleating rays' for $\mathcal{Q}$. We do not know of any way to extend the action of a holomorphic correspondence on $\hat{\mathbb{C}}$ into the interior of the Poincaré disc that would enable us to read off 'pleating coordinates' from the geometric structure of the limit set of the correspondence, but the following is an alternative approach which appears promising. We label the two branches of $\mathcal{F}_{a}$, and for each rational $p / q$ we consider the iterate $\mathcal{F}_{a}^{q}$ corresponding to a 'Sturmian' word $W_{p / q}$ in the two labels. Then we say that $a$ lies on the $p / q$-ray if this iterate has real derivative when applied at whichever of the marked points lies on a leaf of the lamination of $\Omega_{2}$ corresponding to $p / q$ : the size of the derivative can be used as a measure of distance along the ray.

Recall that the patterns $P_{m, n}$ give global laminations of Type 2, that is to say the leaves all have at least one end on the boundary of $\Omega_{2}$. Contracting such a lamination corresponds to moving towards the end of the corresponding ray in $\mathcal{Q}$. Topologically there is no difficulty about contracting the leaves of the corresponding global lamination to points, yielding a topological model for a 'pinched' 

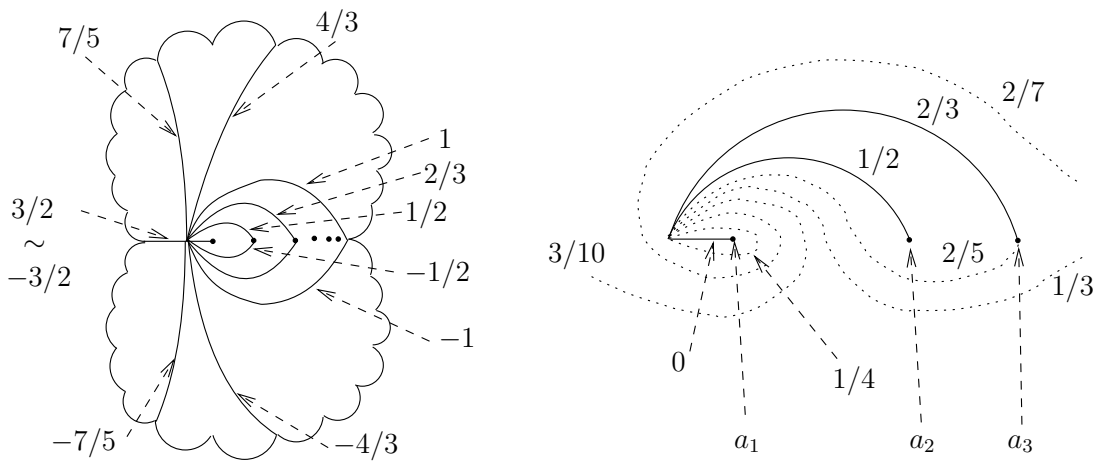

Figure 14. Schematic illustrations of pleating rays on $\mathcal{Q}$. Here region $I$ of Figure 5 has been inverted, to put $a=\infty$ on the inside. The base point of the rays is taken close to $a_{0}$ (where the correspondence is the modular group), and the critical relation parameter values $a_{n}$ are shown as black dots. In the close-up on the right, some of the rays which cross the real axis are indicated: the 1/4-ray returns to the base point (as does the $7 / 12$-ray in the next gap), the $1 / 3$ and $2 / 7$-rays head to the same boundary value as the 1 and -1 -rays (namely $a_{\infty}$ ), and the $3 / 10$-ray ends at the same boundary value as the $3 / 2$ and $-3 / 2$-rays.

correspondence, but there are technical difficulties in performing the contraction holomorphically.

Conjecture 1. (i) For each coprime pair $(m, n)$, with $1<m<n$, the global lamination associated to the pattern $P_{m, n}$ supports a pinching deformation which converges to some $\mathcal{F}_{a_{(m, n)}}$, with $a_{(m, n)}$ on the boundary of $\mathcal{Q}$.

(ii) The values $a_{(m, n)}$ are dense in the boundary of $\mathcal{Q}$.

(iii) The boundary of $\mathcal{Q}$ is a simple closed curve.

To prove part (i) of the conjecture we need to construct an appropriate convergent pinching deformation. However, the leaves of the global lamination end at a dense set in the boundary of $\Omega_{2}$ and to define the support of a pinching deformation we need the leaves to have equivariant neighbourhoods, necessarily extending a small distance into $\Omega_{1}$. While it is unclear how to construct such neighbourhoods in our situation, there is some hope that it may be possible, as equivariant neighbourhoods have been constructed for an analogous problem [2], where $z \rightarrow z^{2}$ rather than $P S L_{2}(\mathbb{Z})$ acts on the other side of the boundary.

In two particular cases we can show directly that the pinched correspondences are indeed holomorphic.

Example 1. Pinching the global lamination associated to $P_{0,1}$.

Topologically pinching either the global lamination associated to $P_{0,1}$, or equally well that associated to $P_{1,0}$, it is easy to see that the resulting pinched correspondence has the dynamics of a mating of $P S L_{2}(\mathbb{Z})$ with the quadratic map $z \rightarrow z^{2}+1 / 4$ in the sense of [4. The pinching splits $\Omega_{2}$ into two topological discs, a left-hand disc which we denote $\Omega_{l}$, and a right-hand disc which we denote 


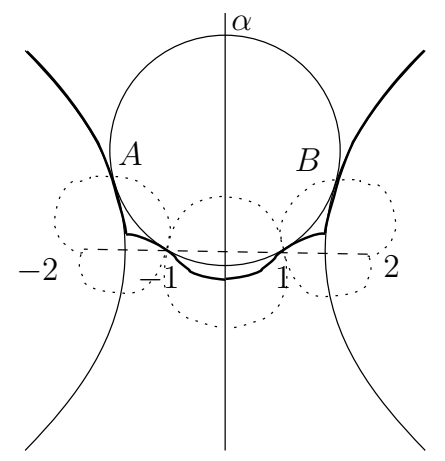

Figure 15. Example 2: A fundamental domain $D_{Q_{1}}$ (bounded by the heavier line) for $\operatorname{Cov}_{0}^{Q_{1}}$, and a fundamental domain $D_{\sigma}$ (bounded by the circle through $\alpha, A, B,-1$ and +1 ) for the involution $\sigma$, such that the pair $\left(D_{Q_{1}}, D_{\sigma}\right)$ satisfy the Klein combination condition. The limit set of the correspondence consists of three topological circles (shown as dotted lines).

$\Omega_{r}$. The pinched correspondence has a 2-to- 1 branch $\Omega_{l} \rightarrow \Omega_{l}$, a 1-to-2 branch $\Omega_{r} \rightarrow \Omega_{r}$, and a 1-to-1 branch $\Omega_{l} \rightarrow \Omega_{r}$, the 2-to-1 branch being topologically conjugate to the quadratic map $q_{1 / 4}: z \rightarrow z^{2}+1 / 4$ on its filled Julia set, and the 1-to-2 branch being conjugate to $\left(q_{1 / 4}\right)^{-1}$. But we already know from [4] that for the parameter value $a=7$, the correspondence $\mathcal{F}_{a}$ in our family realises this mating. It is pictured in the rightmost plot in Figure 4 .

Remark 8. A good way to view correspondences in the quasifuchsian region $\mathcal{Q}$ is to regard them as perturbations of this mating example. Recall that the quadratic map $q_{1 / 4}$ is characterised by the property that it has a parabolic fixed point of multiplier 1. Its critical point, zero, is attracted towards this fixed point but takes infinitely many iterations to get there. As we perturb the value of the parameter away from $a=7$ (where the mating example occurs), the double parabolic fixed point of $\mathcal{F}_{a}$ at which $\Omega_{l}$ meets $\Omega_{r}$ opens up into a 'gateway' through which the orbit of the critical point of $q_{1 / 4}$ on $\Omega_{l}$ can pass and reach the corresponding point of $\Omega_{r}$ in a finite number $n$ of steps, where $n$ goes down as the perturbation increases. This viewpoint can be formalised using Ecalle-Voronin cylinders and Fatou coordinates, and gives an alternative derivation of the existence of the real critical coincidence correspondences $\mathcal{F}_{a_{n}}$ classified in Theorem 1 .

Example 2. Pinching the global lamination associated to $P_{1,1}$.

Our second special case concerns the pattern $P_{1,1}$. The corresponding global lamination (Figure 11) has symmetries which allow us to compute a candidate value of $a$ (on the negative real axis) where the pinched version should occur, and to show that it does indeed occur there.

We work in the $Z$-plane and consider the two images under $\operatorname{Cov}_{0}^{Q_{1}}$ of the imaginary axis. These cross the real axis at the points $Z= \pm \sqrt{3}$. The part of the upper half of the $Z$-plane which lies between these lines is a fundamental domain $D_{Q_{1}}$ for $\operatorname{Cov}_{0}^{Q_{1}}$. For $a$ real and negative the fixed points $\alpha$ and $\beta$ of $\sigma$ lie on the imaginary axis; the parabolic fixed points $A$ and $B$ of $\sigma \circ \operatorname{Cov}_{0}^{Q_{1}}$ are the points where the 


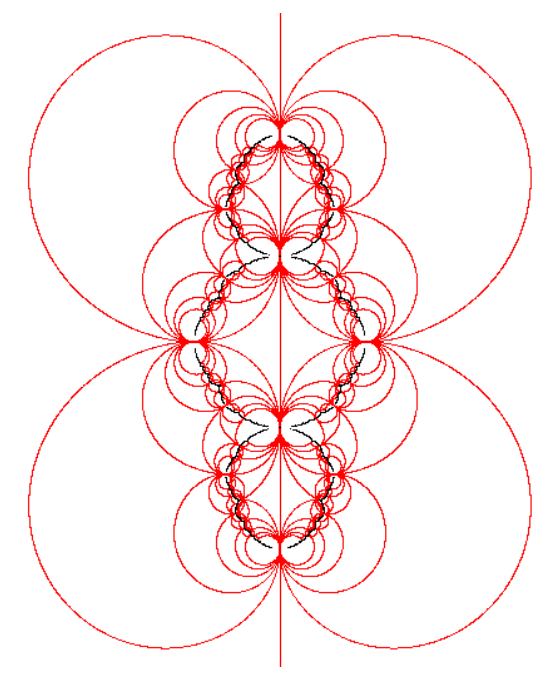

Figure 16. Computer plot of Example $2\left(\mathcal{F}_{a}\right.$ for the value $a=$ $-17-12 \sqrt{ } 2)$. This is plotted in the $z$-plane: the circle through $A$, $\alpha$ and $B$ in the $Z$-plane in Figure 15 has now become the imaginary axis. The images of the imaginary axis are shown in red, and the limit set is plotted in black (due to the limitations of the program the plot is incomplete near the 'pinch points').

circle $C_{\sigma}$ through $\alpha$ and $\beta$ with centre on the imaginary axis touches the boundary of this $D_{Q_{1}}$. Clearly, if $\alpha$ and $\beta$ are both on the upper half of the imaginary axis, the disc $D_{\sigma}$ bounded by $C_{\sigma}$ lies inside $D_{Q_{1}}$, and so $\sigma \circ \operatorname{Cov}_{0}^{Q_{1}}$ is quasifuchsian by a standard Klein combination argument. But for $\beta$ on the imaginary axis just below 0 we can alter the lower boundary of $D_{Q_{1}}$ to an alternative curve invariant under $\operatorname{Cov}_{0}^{Q_{1}}$, as shown in Figure 15, and retain the Klein combination condition. The lowest position of $\beta$ which permits this is when $C_{\sigma}$ passes through the singular points $Z= \pm 1$ (as illustrated). In this configuration $C_{\sigma}$ and $\partial D_{Q_{1}}$ are tangent at four points $A, B, Z=+1$ and $Z=-1$, and the component $\Omega_{2}$ of the regular set of the correspondence $\sigma \circ \operatorname{Cov}_{0}^{Q_{1}}$ is pinched into three topological discs.

It is easy to compute the value of the parameter $a$ at which this configuration occurs, since the circle through $\alpha$ and $\beta$ passes through +1 and -1 if and only if $\beta=1 / \alpha$. Inserting this relation into equation (4) yields $\alpha=(3+2 \sqrt{2}) i$ and $\beta=(2 \sqrt{2}-3) i$. As $a=\alpha / \beta$ we deduce that the configuration occurs at $a=$ $-17-12 \sqrt{2}$. Figure [16] is a computer plot of the images of the imaginary axis under $\mathcal{F}_{a}$ for this value of $a$. Labelling the top, centre, and bottom components of $\Omega_{2}$ for this correspondence as $\Omega_{t}, \Omega_{c}$, and $\Omega_{b}$, one can see that $\mathcal{F}_{a}$ has branches sending $\Omega_{c} \rightarrow \Omega_{t}, \Omega_{c} \rightarrow \Omega_{b}, \Omega_{t} \rightarrow \Omega_{c}, \Omega_{b} \rightarrow \Omega_{c}, \Omega_{t} \rightarrow \Omega_{t}$ and $\Omega_{b} \rightarrow \Omega_{b}$. Each of these branches is bijective and the stabiliser of each component under iterates $\mathcal{F}_{a}^{n}$, $n \in \mathbb{Z}$, is a group. Finally, we note that the points at which the boundaries of the components touch are the critical points $c_{1}$ and $c_{2}$ and that under the appropriate branches of the correspondence these form a (parabolic) 2-cycle.

When pinched topologically, the global laminations associated to all patterns $P_{m, n}$, other than the two examples just considered, pinch $\Omega_{2}$ into a countable 
infinity of discs. Numerical experiments suggest that these examples can all be realised as holomorphic correspondences, and that these lie densely in the boundary of the quasifuchsian region $\mathcal{Q}$ (Conjecture 1). It is an interesting question as to whether one can realise pinchings of irrational laminations and obtain examples where $\Omega_{2}$ is pinched to a dendrite: one would expect examples of this type to provide the remaining points of the boundary of $\mathcal{Q}$.

\section{REFERENCES}

1. S. Bullett, Matings in Holomorphic Dynamics, in Geometry of Riemann Surfaces, edited by Frederick P. Gardiner, Gabino Gonzalez Diez and Christos Kourouniotis, LMS Lecture Notes 368, CUP 2010, 88-119.

2. S. Bullett and P. Haïssinsky, Pinching holomorphic correspondences, Conformal Geometry and Dynamics 11 (2007) 65-89. MR.2314243 (2008e:37044)

3. S. Bullett and W. Harvey, Mating quadratic maps with Kleinian groups via quasiconformal surgery, Electronic Research Announcements of the AMS 6 (2000) 21-30. MR1751536 (2000m:37068)

4. S. Bullett and C. Penrose, Mating quadratic maps with the modular group, Inventiones Math. 115 (1994) 483-511. MR.1262941 (95c:58148)

5. S. Bullett and C. Penrose, Regular and limit sets for holomorphic correspondences, Fund. Math. 167 (2001) 111-171. MR.1816043 (2002d:37068)

6. Linda Keen and Caroline Series, Pleating invariants for punctured torus groups, Topology 43 (2004), 447-491. MR2052972 (2005f:30077)

7. R. Mañé, P. Sad and D. Sullivan, On the dynamics of rational maps, Ann. Sci. Ec. Norm. Sup. (Paris) 16 (1983) 193-217. MR732343 (85j:58089)

8. David Mumford, Caroline Series, and David Wright, Indra's Pearls: the Vision of Felix Klein, CUP 2002. MR1913879 (2003f:00005)

9. M. Samarasinghe, Ph.D. thesis, Queen Mary University of London, 2008.

10. D. Sullivan and W. Thurston, Extending holomorphic motions, Acta Math. 157 (1986) 243258. MR857674 (88i:30033)

School of Mathematical Sciences, Queen Mary University of London, Mile End Road, LONDON E1 4NS, UNITED KingDOM

E-mail address: s.r.bullett@qmul.ac.uk 